\title{
Two-step strongly first-order electroweak phase transition modified FIMP dark matter, gravitational wave signals, and the neutrino mass
}

\author{
Ligong Bian ${ }^{1,2, *}$ and Xuewen $\mathrm{Liu}^{3, \dagger}$ \\ ${ }^{1}$ Department of Physics, Chongqing University, Chongqing 401331, China \\ ${ }^{2}$ Department of Physics, Chung-Ang University, Seoul 06974, Korea \\ ${ }^{3}$ Department of Physics, Nanjing University, Nanjing 210093, China
}

(Received 13 January 2019; published 8 March 2019)

\begin{abstract}
We study the dynamical freeze-in production of dark matter considering the electroweak phase transition history of the Universe. The kinematical thresholds of the decay and scattering processes for dark matter production can be altered by the temperature-dependent thermal masses of particles, which might lead to an enhancement or reduction of the dark matter relic abundance. The second-stage strongly first-order electroweak phase transition (SFOEWPT) triggered by the hidden scalars can be probed at colliders and gravitational wave detectors. Two-step SFOEWPT modified late decay feebly interacting massive particle dark matter is accomplished with a Dirac neutrino mass explanation in the scotogenic model.
\end{abstract}

DOI: 10.1103/PhysRevD.99.055003

\section{INTRODUCTION}

Dark matter (DM), as an essential ingredient of the standard cosmological model, is supported by cosmological observations [1] and can be explained as particles beyond the standard model (SM). The nongravitational property of DM is not known to us [2]. The explanations of the DM motivates the weakly interacting massive particles (WIMPs), where DM weakly interacts with the SM sector but strongly enough to be probed at various direct detection experiments [3-5], indirect detection experiments [6-10], and colliders, such as the LHC and future CEPC, ILC, and FCC-ee. However, the lack of signals of WIMPs at these detectors motivates people to go beyond the WIMP paradigm [11]. In the WIMP paradigm, the DM particles live in a thermal bath at a high temperature through interaction with the SM particles, and the DM relic abundance is produced by the freeze-out mechanism after the DM particle annihilation rate falls below the Universe expansion rate at a temperature around $T_{\mathrm{fo}} \sim m_{\mathrm{DM}} / x_{\mathrm{fo}}$ with $x_{\mathrm{fo}} \sim 26$ [12]. One alternative approach can be the feebly interacting massive particle (FIMP), where the DM particles never enter into thermal equilibrium with the SM plasma due to the superweak or feeble interaction rate between the DM particles and the SM particles [13-15]. In

\footnotetext{
*1gbycl@cqu.edu.cn

†xuewenliu@nju.edu.cn
}

Published by the American Physical Society under the terms of the Creative Commons Attribution 4.0 International license. Further distribution of this work must maintain attribution to the author(s) and the published article's title, journal citation, and DOI. Funded by SCOAP. this paradigm, a basic assumption is that the initial DM abundance (at the reheating epoch) is negligible ${ }^{1}$ and the present DM abundance is produced by the so-called freezein mechanism, through the SM particles (in the thermal bath) decaying or annihilating to the DM particles at a high temperature around $T_{\mathrm{fi}} \sim m_{\mathrm{DM}} / x_{\mathrm{fi}}$ with $x_{\mathrm{fi}} \sim 1[14,15,22]$. There also exist some other nonthermal DM production mechanisms such as incomplete reheating [20], asymmetric reheating [21,23,24], and Dodelson-Widrow mechanism [25], which, however, we do not consider in this work.

In the FIMP scenario, $T_{\mathrm{fi}} \sim \mathcal{O}\left(10^{2}\right) \mathrm{GeV}$ for the weakscale DM, and this temperature is in the ballpark of the electroweak phase transition temperature of the Universe. One can expect that the thermal effects from the electroweak phase transition change the production of the FIMP DM by modifying the kinematical threshold of the DM decay and annihilation processes; recent studies can be found in Refs. [26-28]. ${ }^{2}$ The electroweak phase transition provides an explanation for the electroweak symmetry breaking of the SM at the early Universe, which can be

\footnotetext{
${ }^{1}$ The population of DM for the initial negligible DM abundance is related to the deep understanding of the early Universe. Because of the FIMP, DM never enters into thermal equilibrium, and primordial perturbations in the DM density spectrum would not be washed out and may leave an imprint in the cosmic microwave background (CMB), especially when the FIMP DM is the scalar inflaton $[15,16]$. This feature is supposed to limit the FIMP models [15]. The interaction strength between the inflaton and the DM sector might be bounded by the DM relic density [17-21]. We left the connection of this work with the inflation and the reheating to a separate publication.

${ }^{2}$ For the phase transition modified WIMP scenario, we refer to Ref. [29].
} 
tested by probing the triple Higgs couplings at high-energy hadronic colliders [30] and the future linear colliders, such as CEPC, ILC, and FCC-ee. On the other hand, supposing the electroweak phase transition is a strongly first-order phase transition (SFOEWPT), space-based gravitational wave detectors can probe the gravitational wave signals from the phase transition.

Recently, the hidden sector triggered multistep phase transition aroused people's interest; see Refs. [31-34] for the combined studies of the WIMP DM and the two-step SFOEWPT, wherein the first step is a second-order phase transition, and the second step is a SFOEWPT. There also exist a broad class of well-motivated DM models with more than one hidden sector, wherein the DM particle is one component of the hidden sectors $[35,36]$. In this work, we study the two-step SFOEWPT scenario with the FIMP DM particle being one component of the hidden sectors. The nonzero neutrino mass is well established by oscillation experiments, which requires new physics beyond the SM [37]. ${ }^{3}$ Therefore, it would be inspiring if the neutrino mass could be addressed together with the two-step SFOEWPT modified FIMP DM with the DM being a part of hidden sectors. This constitutes the essential ingredient of this work. The scotogenic model proposed by $\mathrm{Ma}$ [39] falls into the context of the DM models, where the Dirac neutrino mass is radiatively generated with the augmentation of two hidden sectors (including an inert scalar doublet and a scalar singlet). ${ }^{4}$ The DM particles in the model can be the lightest of the heavy neutrinos or the neutral scalars from the dark sector. ${ }^{5}$

This work is organized as follows: We first review the scotogenic model in Sec. II. The phase transition patterns and the implications for FIMP DM production are explored in Sec. III. The DM phenomenology is studied in Sec. IV. The gravitational wave signal predictions and related collider interplay are discussed in Secs. V and VI. We conclude with Sec. VII.

\section{THE SCOTOGENIC MODEL}

We revisit the scotogenic model that generates the Dirac neutrino mass with dark matter at the one-loop level [39]. The Yukawa interactions for the radiative Dirac neutrino mass generation are given by

\footnotetext{
${ }^{3}$ The bounds on the summation of the neutrino masses from cosmology were given recently by Ref. [38].

${ }^{4}$ For the study on WIMP DM as the mixing of the two scalars, we refer to Ref. [40].

${ }^{5}$ Relevant studies within the WIMP paradigm can be found in Ref. [39] and references therein. Reference [41] studied the FIMP realization of DM within the original scotogenic model of Ref. [42], where the Majorana neutrino mass is radiatively generated by the help of an inert scalar sector.
}

$$
\begin{aligned}
\mathcal{L}_{Y}= & f_{\alpha \kappa} \bar{N}_{\kappa}\left(\frac{1-\gamma_{5}}{2}\right)\left(\nu_{\alpha} H_{0}-l_{\alpha} H^{+}\right) \\
& +h_{\kappa \beta} \bar{N}_{\kappa}\left(\frac{1+\gamma_{5}}{2}\right) \nu_{\beta} S+\text { H.c. }
\end{aligned}
$$

where the introduced inert doublet $(\eta)$ together with an additional real singlet scalar $(S)$ are all odd under the $\mathbb{Z}_{2}$ symmetry, and the tree-level potential of the model is

$$
\begin{aligned}
V= & \mu_{\Phi}^{2} \Phi^{\dagger} \Phi+\mu_{\eta}^{2} \eta^{\dagger} \eta+\frac{\mu_{S}^{2}}{2} S^{2}+\lambda_{1}\left(\Phi^{\dagger} \Phi\right)^{2}+\lambda_{2}\left(\eta^{\dagger} \eta\right)^{2} \\
& +\lambda_{3}\left(\Phi^{\dagger} \Phi\right)\left(\eta^{\dagger} \eta\right)+\lambda_{4}\left(\eta^{\dagger} \Phi\right)\left(\Phi^{\dagger} \eta\right) \\
& +\frac{1}{2} \lambda_{5}\left[\left(\eta^{\dagger} \Phi\right)^{2}+\left(\Phi^{\dagger} \eta\right)^{2}\right]+\frac{\lambda_{s}}{4} S^{4}+\lambda_{s \phi} S^{2}\left(\Phi^{\dagger} \Phi\right) \\
& +\lambda_{s \eta} S^{2}\left(\eta^{\dagger} \eta\right)+\mu_{\mathrm{soft}} S\left(\Phi^{\dagger} \eta+\eta^{\dagger} \Phi\right) .
\end{aligned}
$$

In the global minimum of the electroweak (EW) vacuum, the doublets are given as

$\Phi=\left(\begin{array}{c}G^{+} \\ \frac{1}{\sqrt{2}}\left(v+h+i G^{0}\right)\end{array}\right), \quad \eta=\left(\begin{array}{c}H^{+} \\ \frac{1}{\sqrt{2}}\left(H_{0}+i A\right)\end{array}\right)$,

where $\Phi$ develops a vacuum expectation value (VEV) $v=246 \mathrm{GeV}$. The $\mathbb{Z}_{2}$ symmetry of $\eta, S$ remains unbroken in the EW vacuum. Goldstones $G^{+}$and $G^{0}$ are eaten by $W^{ \pm}$ and $Z$ bosons after the spontaneous symmetry breaking (SSB). We consider a $C P$-conserving situation in this work, and all soft masses and scalar quartic couplings in Eq. (2.2) are assumed to be real; the parameter relations and relevant theoretical and experimental constraints are given in Appendix A. In Ref. [43], a one-step SFOEWPT is reachable due to a sizable dimensional-six operator of Higgs after integrating out the heavy scalars in the scalar potential. While in this study we focus on the two-step phase transition, since the dimensional-six operator can be obtained only at the loop level, see Refs. [44,45], which is different from our previous study on mixed scalar WIMP DM [40], where a sizable interaction rate between the SM Higgs sector and the dark scalars is allowed.

In the EW vacuum, the mixing between the singlet and the inert doublet neutral scalars $S, H_{0}$ connects the gauge eigenstates to the mass eigenstates $\chi$ and $H$ by

$$
\left(\begin{array}{c}
S \\
H_{0}
\end{array}\right)=\left(\begin{array}{cc}
\cos \theta & -\sin \theta \\
\sin \theta & \cos \theta
\end{array}\right)\left(\begin{array}{l}
\chi \\
H
\end{array}\right) \text {. }
$$

Here, the mixing angle is

$$
\theta=\frac{1}{2} \tan ^{-1}\left(\frac{2 v \mu_{\mathrm{soft}}}{\tilde{M}_{S}^{2}-\tilde{M}_{H_{0}}^{2}}\right),
$$

with $\tilde{M}_{S}^{2}=2 \mu_{S}^{2}+\lambda_{s \phi} v^{2}$ and $\tilde{M}_{H_{0}}^{2}=\mu_{\eta}^{2}+\lambda_{L} v^{2}$. For parameter relations between mass eigenstates and gauge eigenstates, see Appendix A 1. 


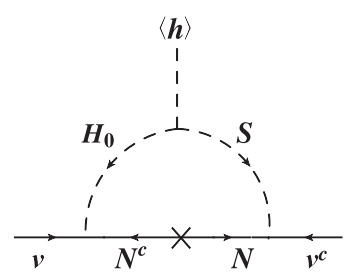

FIG. 1. The neutrino mass generation in the scotogenic model.

From Eq. (2.1), one gets the one-loop Dirac neutrino mass:

$$
\begin{aligned}
\left(M_{\nu}\right)_{\alpha \beta}= & \frac{\sin 2 \theta}{32 \sqrt{2} \pi^{2}} \sum_{\kappa} f_{\alpha \kappa} h_{\kappa \beta} m_{N_{\kappa}} \\
& \times\left[\frac{m_{\chi}^{2}}{m_{\chi}^{2}-m_{N_{\kappa}}^{2}} \log \frac{m_{\chi}^{2}}{m_{N_{\kappa}}^{2}}-\frac{m_{H}^{2}}{m_{H}^{2}-m_{N_{\kappa}}^{2}} \log \frac{m_{H}^{2}}{m_{N_{\kappa}}^{2}}\right],
\end{aligned}
$$

with the Feynman diagram given in Fig. 1.

For the scotogenic model proposed in Ref. [42], the dangers of flavor-changing charged-lepton radiative decays can be avoided by assuming a superweak Yukawa interaction of $f_{\alpha \kappa}$, which prefers a FIMP DM $[14,41,46]$. Indeed, a superweak interaction of $N_{1}$ with scalars leads to the decoupling of $N_{1}$ from the neutrino mass matrix, and there are only two light neutrinos acquire nonzero masses; see Ref. [41]. For the Dirac neutrino mass generation in the scotogenic model proposed by Ref. [39], the extra scalars are introduced to accommodate the second Yukawa interaction of Eq. (2.1). There is no problem of the flavorchanging charged-lepton radiative decays. In this work, we would explore the $N_{1}$ as the FIMP DM, in which case the $N_{1}$ decouples from the neutrino mass matrix. Furthermore, the smallness of the neutrinos masses can be ensured by the smallness mixing between $S$ and $H_{0}$ in the model, which naturally leads to the production of $\chi$ through the freeze-in mechanism, which then decay to the DM particle $N_{1}$ after the freeze-in. This study applies to the global $U(1)_{B-L}$ scenario of Ref. [39] and the millicharge situation of the gauged $U(1)_{B-L}$ scenario.

\section{THE ELECTROWEAK PHASE TRANSITION DYNAMICS}

Supposing $H, A, H^{ \pm}$, and $S$ are all much heavier than the SM-like Higgs, one can safely integrate out the dark scalars, and therefore the tree-level potential with dimensional-six operators is given by

$$
V_{0}=\mu_{\Phi}^{2}|\Phi|^{2}+\lambda|\Phi|^{4}+c_{6}|\Phi|^{6},
$$

with the $c_{6}$ mainly coming from the one-loop level, given by Refs. [44,45], respectively, for the dark scalar and inert doublet cases. Therefore, to obtain a strongly first-order phase transition with a one-step pattern, a relatively larger quartic coupling is required [44]. This may result in the perturbativity problem $[32,33,44,45]$ even at model phenomenologically valid energy scales. Another reason that motivates us to focus on the two-step pattern phase transition is to account for the production of scalar $\chi$ and the DM $N_{1}$ through the freeze-in mechanism.

\section{A. Electroweak phase transition types and vacuum structures}

First, we analyze the vacuum structure at zero temperature, which is highly related with the possible phase transition types, i.e., one step and two step. The tree-level scalar potential of the classical fields is given by

$$
\begin{aligned}
V_{0}\left(h, H_{0}, S\right)= & \frac{\mu_{\Phi}^{2}}{2} h^{2}+\frac{\mu_{\eta}^{2}}{2} H_{0}^{2}+\frac{\mu_{S}^{2}}{2} S^{2}+\frac{\lambda_{1}}{4} h^{4} \\
& +\frac{1}{4}\left(\lambda_{3}+\lambda_{4}+\lambda_{5}\right) h^{2} H_{0}^{2}+\frac{\lambda_{2}}{4} H_{0}^{4} \\
& +\frac{1}{2} \lambda_{s \phi} h^{2} S^{2}+\frac{1}{2} \lambda_{s \eta} H_{0}^{2} S^{2}+\frac{\lambda_{s}}{4} S^{4} \\
& +\mu_{\text {soft }} h H_{0} S .
\end{aligned}
$$

In the space of $\left(h, H_{0}, S\right)$, the minimization conditions

$$
\begin{aligned}
\frac{\partial}{\partial h} V_{0}\left(h, H_{0}, S\right) & =0, \quad \frac{\partial}{\partial H_{0}} V_{0}\left(h, H_{0}, S\right)=0, \\
\frac{\partial}{\partial S} V_{0}\left(h, H_{0}, S\right) & =0
\end{aligned}
$$

give rise to the possible minima of the potential localized at

$$
\begin{gathered}
\left( \pm \sqrt{-\mu_{\Phi}^{2} / \lambda_{1}}, 0,0\right), \quad\left(0, \pm \sqrt{-\mu_{\eta}^{2} / \lambda_{2}}, 0\right), \\
\left(0,0, \pm \sqrt{-\mu_{S}^{2} / \lambda_{s}}\right), \\
\left(0, \pm \sqrt{\left(\mu_{S}^{2} \lambda_{s \eta}-\mu_{I}^{2} \lambda_{s}\right) /\left(\lambda_{2} \lambda_{2}-\lambda_{s \eta}^{2}\right)},\right. \\
\left.\sqrt{\left(\mu_{\eta}^{2} \lambda_{s \eta}-\mu_{s}^{2} \lambda_{2}\right) /\left(\lambda_{2} \lambda_{2}-\lambda_{s \eta}^{2}\right)}\right) .
\end{gathered}
$$

We note that the possible minima where $h$ and $S$ (or/and $H_{0}$ ) have VEVs is precluded, because both the inert doublet and the real singlet scalar are odd under the $\mathbb{Z}_{2}$ symmetry. The EW vacuum localized at $\left( \pm \sqrt{-\mu_{\Phi}^{2} / \lambda_{1}}, 0,0\right)$ should be the global minimum, which ensures the possibility of the phase transition type of $O \rightarrow C$ in Fig. 2. There also could be three

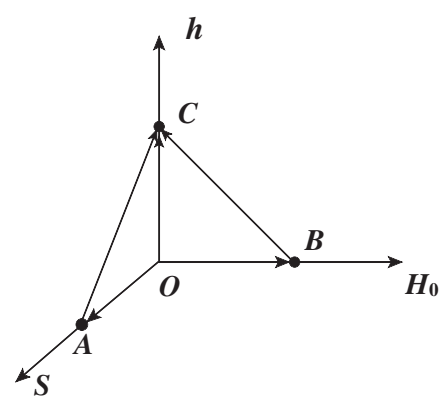

FIG. 2. The phase transition patterns. 

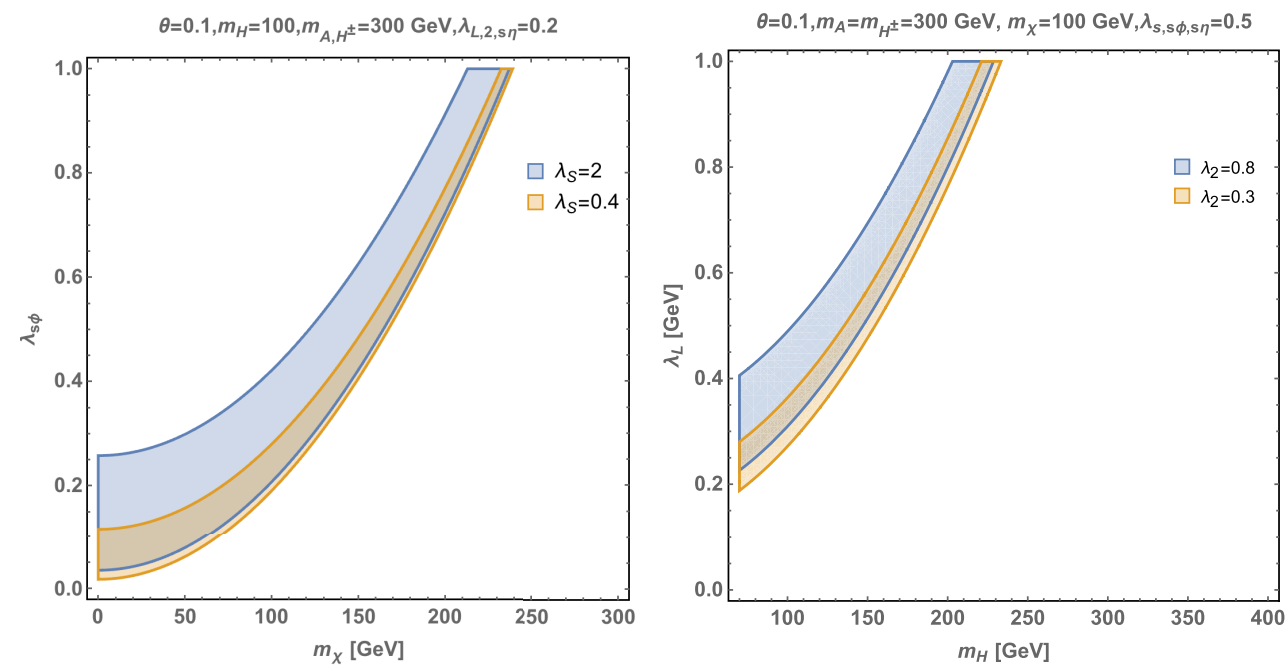

FIG. 3. Two-step patterns of $O \rightarrow A \rightarrow C$ (left) and $O \rightarrow B \rightarrow C$ (right).

other local minima in the direction of $\left(0,\left\langle H_{0}\right\rangle, 0\right),(0,0,\langle S\rangle)$ for the case where the mixing of $H_{0}, S$ is negligible, and in the subspace of $\left(0,\left\langle H_{0}\right\rangle,\langle S\rangle\right)$ when the mixing of $H_{0}, S$ is non-negligible, which, however, is not favored by the neutrino mass generation. To ensure the EW vacuum to be the global vacuum, the following conditions need to be fulfilled:

$$
\begin{gathered}
\Delta V_{0}(h, S) \equiv V_{0}(0,0, S)-V_{0}(h, 0,0)>0, \\
\Delta V_{0}\left(h, H_{0}\right) \equiv V_{0}\left(0, H_{0}, 0\right)-V_{0}(h, 0,0)>0,
\end{gathered}
$$

which result in the following two bounds on the parameter spaces:

$$
\lambda_{s} \mu_{\Phi}^{4}>\lambda_{1} \mu_{s}^{4}, \quad \lambda_{2} \mu_{\Phi}^{4}>\lambda_{1} \mu_{\eta}^{4} .
$$

In the spirit of the gauge invariant $[34,47]$, the finitetemperature potential can be described by Eq. (3.2) with the substitutions of $\mu_{i}^{2} \rightarrow \mu_{i}^{2}(T)$, where

$$
\begin{aligned}
\mu_{\Phi}^{2}(T) & =\mu_{\Phi}^{2}+c_{\Phi} T^{2}, \\
c_{\Phi} & =\frac{6 \lambda_{1}+2 \lambda_{3}+\lambda_{4}+\lambda_{s \phi}}{12}+\frac{3 g^{2}+g^{\prime 2}}{16}+\frac{y_{t}^{2}}{4}, \\
\mu_{\eta}^{2}(T) & =\mu_{\eta}^{2}+c_{\eta} T^{2}, \\
c_{\eta} & =\frac{6 \lambda_{2}+2 \lambda_{3}+\lambda_{4}+\lambda_{s \eta}}{12}+\frac{3 g^{2}+g^{\prime 2}}{16}, \\
\mu_{S}^{2}(T) & =\mu_{S}^{2}+c_{S} T^{2}, \quad c_{S}=\frac{\lambda_{S}}{4}+\frac{\lambda_{s \phi}+\lambda_{s \eta}}{12} .
\end{aligned}
$$

Here, we neglected all other Yukawa couplings apart from the top quark Yukawa coupling $y_{t}$. All three directions of $h$, $H_{0}$, and $S$ can induce minima due to the thermal corrections at a finite temperature depending on these scalar quartic couplings. ${ }^{6}$

The two-step phase transition can occur through $O \rightarrow$ $B \rightarrow C$ (see Fig. 2) when the vacuum along the direction of $H_{0}$ appears earlier than that of the $h$ direction, which corresponds to the parameter constraints of

$$
\begin{array}{ll}
c_{\Phi}>0, & c_{\eta}>0, \quad \mu_{\Phi}<0, \\
\mu_{\eta}<0, & \mu_{\Phi}^{2} c_{\eta}>\mu_{\eta}^{2} c_{\Phi},
\end{array}
$$

or occurs through $O \rightarrow A \rightarrow C$ (see Fig. 2) when the $Z_{2}$ symmetry in the direction of $S$ breaks earlier than the SSB of the EW vacuum in the direction of $h$, which results in

$$
\begin{array}{ll}
c_{\Phi}>0, & c_{S}>0, \quad \mu_{\Phi}^{2}<0, \\
\mu_{S}^{2}<0, & \mu_{\Phi}^{2} c_{S}>\mu_{S}^{2} c_{\Phi} .
\end{array}
$$

In this work, we study the above three patterns of phase transition. For the one-step pattern, the phase transition can occur directly from the EW symmetry and $Z_{2}$ symmetry phase to the EW symmetry broken phase with $Z_{2}$ symmetry. For the two-step pattern of $O \rightarrow A \rightarrow C$, the parameter spaces are restricted by Eqs. (3.7) and (3.12); see the left plot in Fig. 3. For the two-step pattern $O \rightarrow B \rightarrow C$, Eqs. (3.7) and (3.11) restrict the parameters as shown in the right panel in Fig. 3 .

The bounce configuration of the nucleation bubble [the bounce configuration of the multifields that connects the EW broken vacuum ( $h$ vacuum, true vacuum) and the false vacuum-here it can be the $Z_{2}$ broken vacuum $H_{0}(S)$, the vacuum for the two-step scenarios] can be obtained by extremizing

\footnotetext{
${ }^{6}$ If one obtains the minimum along the direction of $S$ at finite temperature, the last term of Eq. (2.2) would induce the term of $\mu_{\text {soft }}\langle S\rangle_{T}\left(\Phi^{\dagger} \eta+\eta^{\dagger} \Phi\right)$.
} 


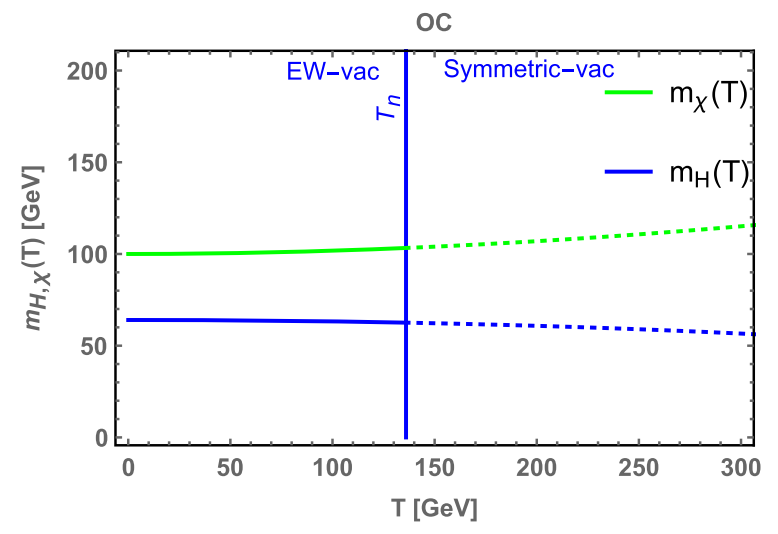

FIG. 4. Thermal masses for the pattern $O \rightarrow C$.

$$
S_{3}(T)=\int 4 \pi r^{2} d r\left[\frac{1}{2}\left(\frac{d \phi_{b}}{d r}\right)^{2}+V\left(\phi_{b}, T\right)\right]
$$

through solving the equation of motion for $\phi_{b}$ (it is $h$ and $H_{0} / S$ for two-step scenarios),

$$
\frac{d^{2} \phi_{b}}{d r^{2}}+\frac{2}{r} \frac{d \phi_{b}}{d r}-\frac{\partial V\left(\phi_{b}\right)}{\partial \phi_{b}}=0,
$$

with the boundary conditions of

$$
\lim _{r \rightarrow \infty} \phi_{b}=0,\left.\quad \frac{d \phi_{b}}{d r}\right|_{r=0}=0 .
$$
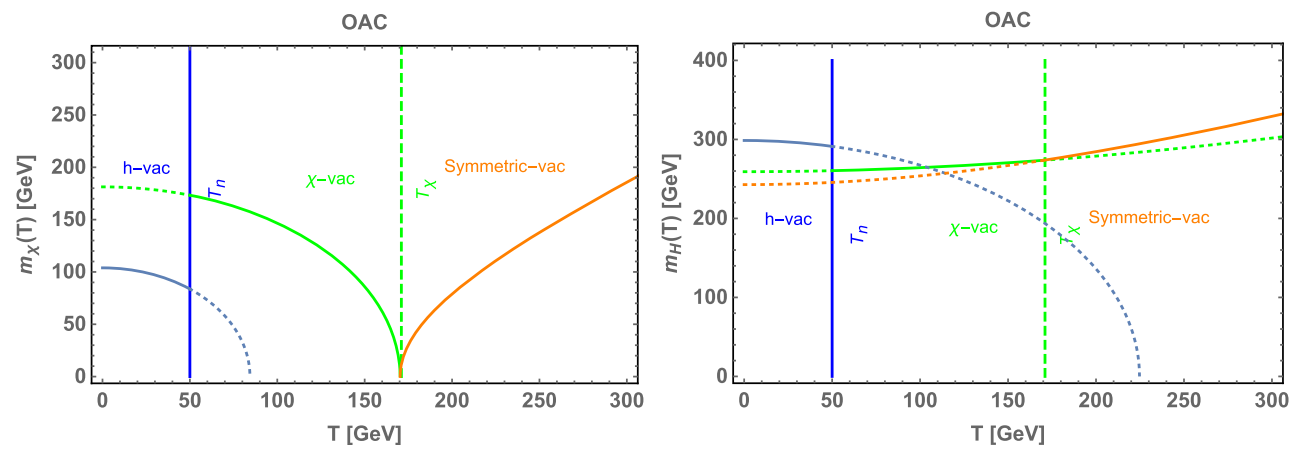

FIG. 5. Thermal masses for the phase transition pattern $O \rightarrow A \rightarrow C$.
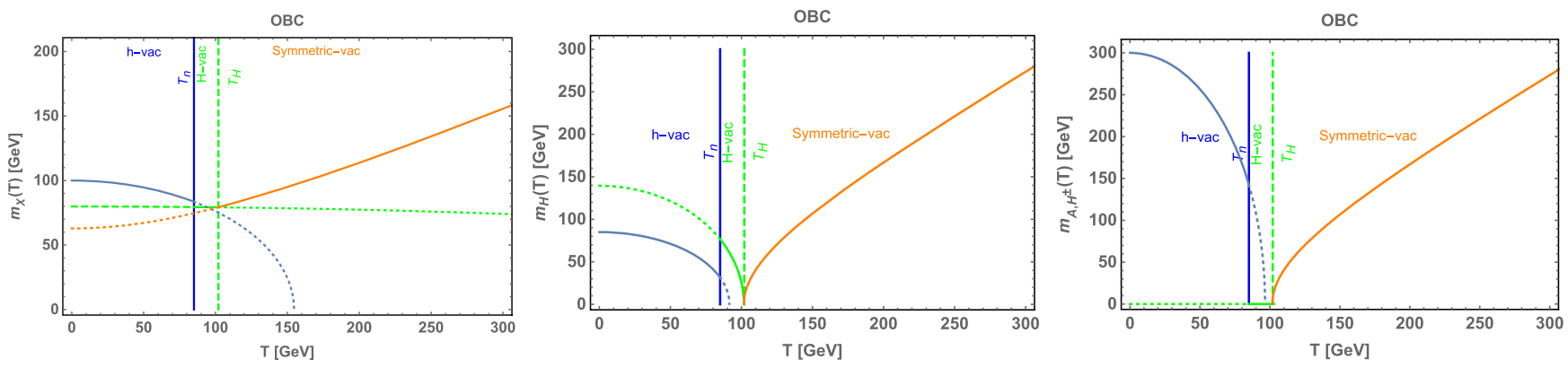

FIG. 6. Thermal masses for the phase transition pattern $O \rightarrow B \rightarrow C$. 
parameters chosen as the bottom-right panel in Fig. 12 and assuming a negligible small $\theta$ ). Therefore, one can expect that the kinematic threshold of the decay or inverse decay and the scattering processes that contribute to the DM production can be different from the traditional FIMP DM calculations. More specifically, the mass splitting between $m_{\chi, A, H, H^{ \pm}}(T)$ and $m_{N_{1}}$ is dynamical and can be vastly different from the zero temperature case in a long time duration, which may lead to an enormous difference of DM relic abundance between the thermal modified calculation and the traditional calculation without taking into account the thermal mass. For the case of $\chi$ producing through the freeze-in mechanism, the $\lambda_{s \phi, s \eta}$ should also be negligible, with $m_{\chi}(T) \approx m_{\chi}$.

\section{DM PHENOMENOLOGY}

For the DM relic density calculation, the accumulated relic abundance of the previous temperature duration will be taken as the initial abundance of the next temperature duration. The DM relic abundance accumulated in the symmetry phase can be significant depending on the reheating temperature, which is taken as the initial condition of the calculation of FIMP where the entropy normalized number density is assumed to be null. For the DM number density calculation with a phase transition, we use the thermal corrected mass to replace the physical mass at zero temperature in the decay and scattering processes.

\section{A. FIMP $N_{1}$ DM}

In the following, we study the case of $m_{\chi, H, H^{ \pm}, A}>m_{N_{1}}$ without mass degeneracy. The relevant study can be found in Ref. [41]; here the difference is that we have an additional decay of $\chi \rightarrow N \nu$. As in Ref. [41], the $2 \leftrightarrow 2$ process is highly suppressed in the situation with phase transition pattern $O \rightarrow A \rightarrow C$, and we therefore focus on the decay or inverse decay of $\chi \rightarrow N \nu$ dominated FIMP DM production. We first study the freeze-in contribution to $N_{1}$ production; its production will be dominated by the decays of the scalars $\left(H, A, H^{ \pm}, \chi\right)$ while they are in equilibrium with the

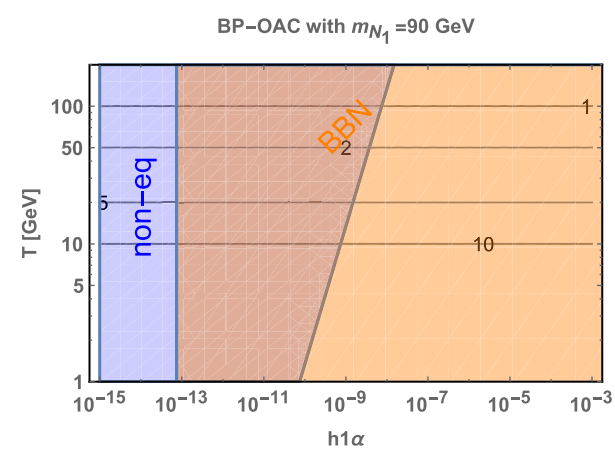

thermal bath. The $N_{1}$ yield for the $O \rightarrow A \rightarrow C$ scenario, $Y_{N_{1}}(T)=n_{N_{1}}(T) / s(T)$, can be computed by solving the following Boltzmann equation [14]:

$$
\frac{d Y_{N}}{d x}=\frac{1}{s x H}\left(\frac{g_{X} m_{X}^{2} m_{N} \Gamma_{X \rightarrow N \nu / \ell}}{2 \pi^{2} x}\right) K_{1}\left(\frac{m_{X}}{m_{N}} x\right),
$$

where $s$ is the entropy density of the Universe and $H(T)$ is the expansion rate of the Universe at a given temperature, with $X=H, A, H^{ \pm}, \chi$, and $\ell$ being a SM lepton. In this equation, $K_{1}(x)$ is the Bessel function of the second kind, and $g_{X}$ is the number of internal degrees of freedom of particle $X$. Specifically, $g_{H, A, H^{+}, H^{-}, \chi}=1$. The decay rates that enter into $\Gamma\left(X \rightarrow N_{1} \ell\right)$ are calculated as

$$
\Gamma\left(H \rightarrow N_{1} \bar{\nu}_{\alpha}\right)=\frac{\left(m_{H}^{2}-m_{N_{1}}^{2}\right)^{2}}{32 \pi m_{H}^{3}}\left(f_{1 \alpha}^{2} \cos ^{2} \theta+2 h_{1 \alpha}^{2} \sin ^{2} \theta\right),
$$

$$
\begin{gathered}
\Gamma\left(\chi \rightarrow N_{1} \bar{\nu}_{\alpha}\right)=\frac{\left(m_{\chi}^{2}-m_{N_{1}}^{2}\right)^{2}}{32 \pi m_{\chi}^{3}}\left(2 h_{1 \alpha}^{2} \cos ^{2} \theta+f_{1 \alpha}^{2} \sin ^{2} \theta\right), \\
\Gamma\left(A \rightarrow N_{1} \bar{\nu}_{\alpha}\right)=\frac{\left(m_{A}^{2}-m_{N_{1}}^{2}\right)^{2}}{32 \pi m_{A}^{3}} f_{1 \alpha}^{2}, \\
\Gamma\left(H^{+} \rightarrow N_{1} \bar{\ell}_{\alpha}\right)=\frac{\left(m_{H^{+}}^{2}-m_{N_{1}}^{2}\right)^{2}}{32 \pi m_{H^{+}}^{3}} f_{1 \alpha}^{2}
\end{gathered}
$$

First, the requirement that the $N_{1}$ does not reach thermal equilibrium, i.e., $\Gamma\left(X \rightarrow N_{1} \nu\right)<H(T)$, sets the upper limits on the Yukawa couplings $h_{1 \alpha}$, and the lower limits are set by the big-bang nucleosynthesis (BBN) requirements [51]. At the $\mathrm{BBN}$ epoch, the finite temperature effects are negligible. We note that the increase of DM mass $m_{N_{1}}$ means a larger parameter spaces allowed by the nonequilibrium conditions and the BBN, as shown in Fig. 7. As for the Yukawa coupling of $f_{1}$, one needs to worry about the disturbation to the BBN

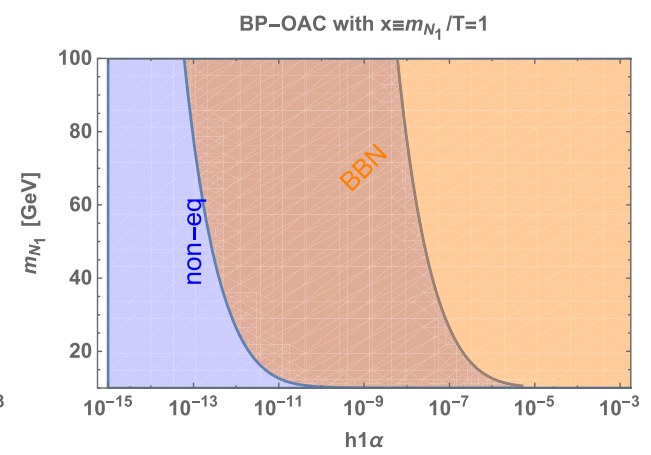

FIG. 7. The decoupling condition bounds on the $h_{1 \alpha}$ for the pattern of $O \rightarrow A \rightarrow C$ correspond to Fig. 12. The $z=m_{N_{1}} / T$ is shown by black lines. 
or CMB by the decay of dark scalars to $N_{1}$, because all these dark scalars are in equilibrium before $T_{\mathrm{fo}} \sim m_{A, H, H^{ \pm}} / 26$ and these particles' number density quickly drops to almost zero after $T_{\text {fo }}$.

Different from the $O \rightarrow A \rightarrow C$ benchmark, in the benchmark $O \rightarrow B \rightarrow C$, we have the contribution of the $2 \leftrightarrow 2$ process $\gamma H^{ \pm} \leftrightarrow \ell N_{1}$ dominating over the decay or inverse decay process $H / A / H^{ \pm} \leftrightarrow N \ell$ when one does not include the thermal mass correction. During the epoch between $T_{H}$ (the first-stage second-order phase transition of $Z_{2}$ phase) and $T_{n}$ (the second-stage first-order phase transition of EW phase), we have a null value for $m_{H^{ \pm}}(T)$, where the contribution of the $H / A \rightarrow N \ell$ process can dominate over the $2 \leftrightarrow 2$ process contribution to the DM production depending on if the kinematical threshold is allowed. Before the $Z_{2}$ phase is broken $\left(T>T_{H}\right)$, i.e., in the symmetric phase, and after the EW phase is broken $\left(T<T_{n}\right)$, the $2 \leftrightarrow 2$ process can dominate over the $H / A \rightarrow N \ell$ process once the kinematical threshold is allowed. The Boltzmann equation for this scenario is

$$
\begin{aligned}
\frac{d Y_{N}}{d x}= & \frac{1}{s x H}\left[\left(\frac{g_{X} m_{X}^{2} m_{N} \Gamma_{X \rightarrow N \nu / \ell}}{2 \pi^{2} x}\right) K_{1}\left(\frac{m_{X}}{m_{N}} x\right)\right. \\
& \left.+\frac{g_{\gamma} g_{H^{ \pm}}}{32 \pi^{4}} \frac{m_{N}}{x} \int_{m_{H^{ \pm}}^{2}}^{\infty} d s 4 p_{\gamma H^{ \pm}}^{2} \sigma_{\gamma H^{ \pm} \rightarrow \ell N} K_{1}\left(\frac{x \sqrt{s}}{m_{N}}\right)\right],
\end{aligned}
$$

where $p_{\gamma H^{ \pm}}=\left(s-m_{H^{ \pm}}^{2}\right) / 2 \sqrt{s}$, and the cross section of $\sigma_{\gamma H^{ \pm} \rightarrow \ell N}$ is given in Appendix C.

In Fig. 8, we show the entropy normalized number density $Y_{N_{1}}$ as a function of the temperature for fixed $m_{N_{1}}$. There, the mixing angle effects are negligible. In the $O \rightarrow A \rightarrow C$ and
$O \rightarrow B \rightarrow C$ scenarios, all dark scalars should live in the thermal bath to avoid disturbing the BBN. In the $O \rightarrow B \rightarrow$ $C$ scenario, the dominant channel is the scattering process, and, therefore, the results do not rely on the mixing angle. The curve "with(out) PT" stands for the results with(out) taking into account the thermal effects. In this situation, all the dark scalars live in the thermal bath before the freeze-out due to the sizable DM-SM couplings. The left panel in Fig. 8 $(O \rightarrow A \rightarrow C)$ is obtained by solving Eq. (4.1) with the thermal masses as a function of the temperature shown in Fig. 5. The right panel in Fig. $8(O \rightarrow B \rightarrow C)$ is obtained by solving Eq. (4.6) (all the thermal masses adopted here are the same as Fig. 6). The departure of the "with PT" yield $Y_{N_{1}}(x)$ from the "without PT" yield is noticeable; this is because the kinematical thresholds for the decay and scattering process are dynamical during the temperature evolution. In particular, the thermal mass of $A, H^{ \pm}$is mostly smaller than the corresponding zero temperature masses within the temperature epoch under study. The two plateau behaviors of the solid curves reflect if the thermal masses reach the threshold of the DM production processes during the two-stage phase transition.

\section{B. Freeze-in $N_{1}$ augmented by late decay of FIMP $\chi$}

In this section, we study the case that $\mathrm{DM} N_{1}$ is partially generated by the freeze-in mechanism and partially generated by a late decay of FIMP $\chi$, in the phase transition pattern $O \rightarrow B \rightarrow C$. When the Yukawa couplings $f_{1 \alpha} \sim$ $h_{1 \alpha} \ll \mathcal{O}(1)$ and $\lambda_{s \phi, s \eta}, \mu_{\text {soft }} \ll 1$, both $\chi$ (in this case, mostly the $S$ component) and $N_{1}$ never reach thermal equilibrium (both are FIMPs). Then the coupled Boltzmann equations are

$$
\begin{aligned}
\frac{d Y_{\chi}}{d x}= & \frac{1}{s x H}\left[\left(\frac{g_{H^{ \pm}(A)} m_{H^{ \pm}(A)}^{2} m_{\chi}}{2 \pi^{2} x}\right) \Gamma_{H^{ \pm}(A) \rightarrow \chi W^{\mp}(Z)} K_{1}\left(\frac{m_{H^{ \pm}(A)}}{m_{\chi}} x\right)-s \Gamma\left(\chi \rightarrow N_{1} \nu_{\alpha}\right) Y_{\chi}\right], \\
\frac{d Y_{N}}{d x}= & \frac{1}{s x H}\left[\left(\frac{g_{H^{ \pm}, A, H} m_{H^{ \pm}, A, H}^{2} m_{N} \Gamma_{H^{ \pm}, A, H \rightarrow N \ell}}{2 \pi^{2} x}\right) K_{1}\left(\frac{m_{H^{ \pm}, A, H}}{m_{N}} x\right)+s \Gamma\left(\chi \rightarrow N_{1} \nu_{\alpha}\right) Y_{\chi}\right. \\
& \left.+\frac{g_{\gamma} g_{H^{ \pm}}}{32 \pi^{4}} \frac{m_{N}}{x} \int_{m_{H^{ \pm}}^{2}}^{\infty} d s 4 p_{\gamma H^{ \pm}}^{2} \sigma_{\gamma H^{ \pm} \rightarrow \ell N} K_{1}\left(\frac{x \sqrt{s}}{m_{N}}\right)\right] .
\end{aligned}
$$
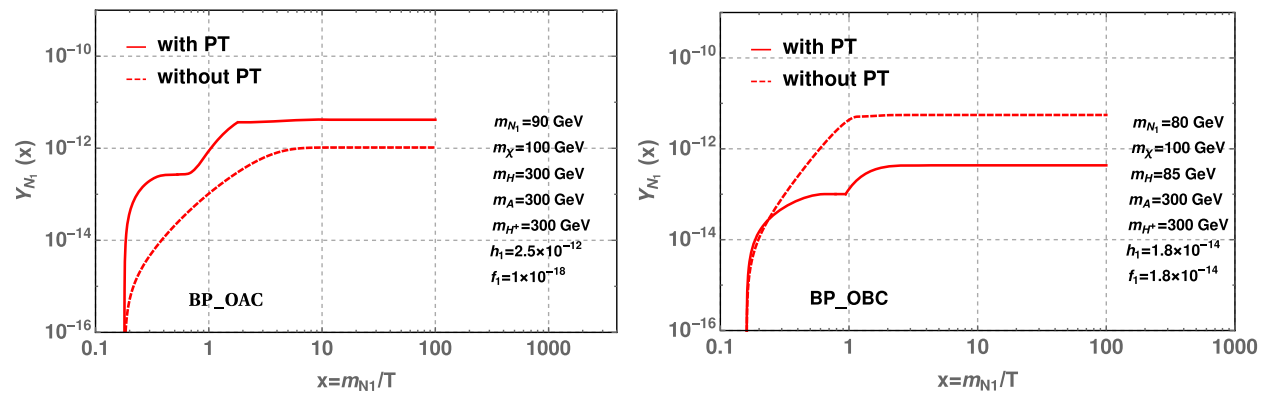

FIG. 8. Relic density of the dark matter $N_{1}$ in the benchmark model of $O \rightarrow A \rightarrow C$ (left panel) and $O \rightarrow B \rightarrow C$ (right panel) with and without thermal effects; $f_{1}$ and $h_{1}$ represent the Yukawa couplings of the first generation of $N_{1}$. 
The decay widths are given as follows:

$$
\begin{gathered}
\Gamma\left(H^{ \pm} \rightarrow W^{ \pm} \chi\right)=\frac{e^{2}\left(m_{H^{+}}^{4}+\left(m_{\chi}^{2}-m_{W}^{2}\right)^{2}-2 m_{H^{+}}^{2}\left(m_{\chi}^{2}+m_{W}^{2}\right)\right)^{3 / 2} \sin ^{2} \theta}{64 \pi m_{W}^{2} \sin ^{2} \theta_{w} m_{H^{+}}^{3}}, \\
\Gamma(A \rightarrow Z \chi)=\frac{e^{2}\left(m_{A}^{4}+\left(m_{\chi}^{2}-m_{Z}^{2}\right)^{2}-2 m_{A}^{2}\left(m_{\chi}^{2}+m_{Z}^{2}\right)\right)^{3 / 2} \sin ^{2} \theta}{64 \pi m_{Z}^{2} \cos ^{2} \theta \sin ^{2} \theta_{w} m_{A}^{3}}, \\
\Gamma\left(\chi \rightarrow N_{1} \nu_{\alpha}\right)=\frac{\left(m_{\chi}^{2}-m_{N_{1}}^{2}\right)^{2}}{64 \pi m_{\chi}^{3}}\left(2 h_{1 \alpha}^{2} \cos ^{2} \theta+f_{1 \alpha}^{2} \sin ^{2} \theta\right) .
\end{gathered}
$$

The $\chi$ particle mostly comes from the inert sectors decay as given by Eqs. (4.8) and (4.9), and the decoupling condition is given by $\Gamma\left(H^{ \pm} / A \rightarrow \chi W^{ \pm} / Z\right)<H(T)$. The left panel in Fig. 9 depicts that the combination of the nonequilibrium condition and BBN bounds the mixing angle $10^{-11}<\theta<10^{-6}$ depending on the temperature. To obtain the correct magnitude of the neutrino masses, as mentioned in Sec. II, the mixing angle cannot be infinitely small. For the $\theta$ allowed by the nonequilibrium conditions and the BBN, the Yukawa couplings of $f_{2,3}$ and $h_{2,3}$ and the heavy neutrino masses are restricted, as indicated by

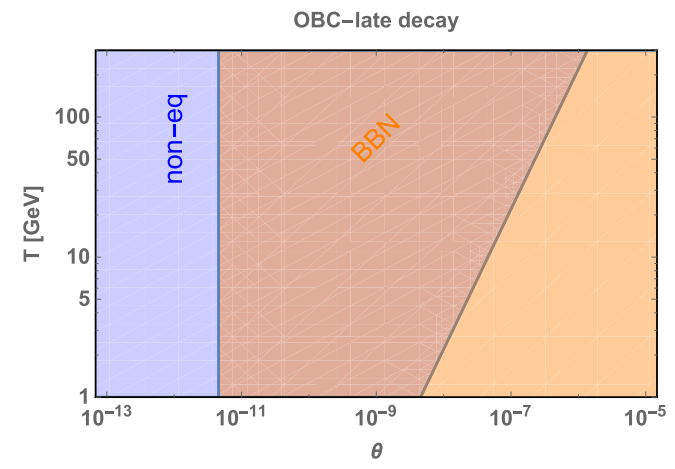

Eq. (2.6). So we illustrate the constraints in the right panel in Fig. 9; the mixing angle $\theta$ and the heavy neutrinos mass are restricted into narrow regions for typical values of the Yukawa couplings $f_{\alpha 2,3}$ and $h_{2,3 \beta}$.

We consider that the $\chi$ particle mostly comes from the decay of $H^{ \pm}$, and then the late decay $\chi \rightarrow N_{1} \nu$ dominantly contributes to the relic density of $N_{1}$. Before that, the contribution of the annihilation process dominates the production of $N_{1}$, as depicted in the right panel in Fig. 8. In the left panel in Fig. 10, we show the without PT case without taking into account the thermal mass, and

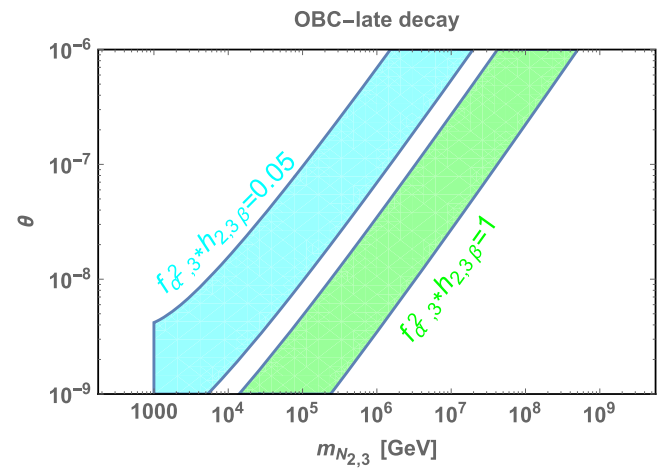

FIG. 9. Left: Parameter spaces of $\theta$ allowed by nonequilibrium conditions and BBN of the late decay of FIMP $\chi$-dominated DM production in the scenario of $O \rightarrow B \rightarrow C$. Right: The neutrino mass allowed parameter spaces of $\theta$ and $m_{N_{2,3}}$ from Eq. (2.6); here we set the range of $10^{-2}-10^{-1} \mathrm{eV}$ for the neutrino mass.
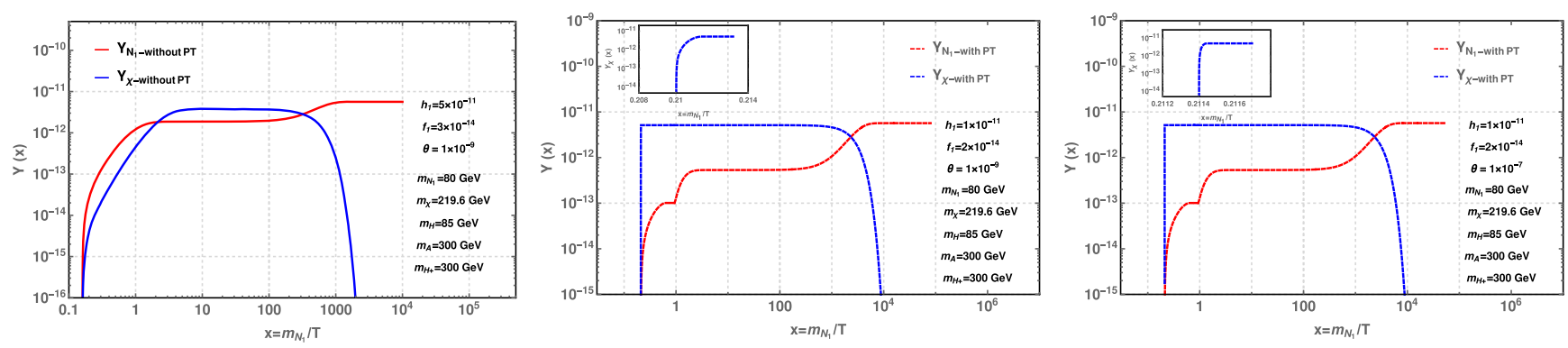

FIG. 10. Relic density of the dark matter $N_{1}$ in the benchmark model of $O \rightarrow B \rightarrow C$ without thermal effects (left) and with thermal effects by taking $\theta=10^{-9}$ (left and middle panels) and $\theta=10^{-7}$ (right panel). The insets illustrate the rapid growth of $Y_{\chi}$ at the early stage. The same as Fig. 8, the $f_{1}$ and $h_{1}$ are the Yukawa couplings of the first-generation $N_{1}$. 
the decay $H^{ \pm} \rightarrow \chi W^{ \pm}$is always active during the production of the $\chi$ particle. For the case with the thermal effect labeled as with PT in the right two panels in Fig. 10, the thermal masses $m_{H, A, H^{ \pm}}(T)$ are adopted, and $m_{\chi}(T) \approx m_{\chi}$, since here we need to assume negligibly small $\lambda_{s \eta, s \phi}, \theta$ to ensure $\chi$ does not enter into equilibrium. The kinematical threshold of $H^{ \pm} \rightarrow \chi W^{ \pm}$can open only at the pretty early stage at a high temperature, which leads to a sharp increase of $Y_{\chi}$ at the beginning. For the cases with the thermal effects, the correct DM relic density highly relies on the reheating temperature due to the modification of the kinematical threshold by the thermal masses; we plot the middle and the right panels in Fig. 10 to illustrate this situation. In comparison with the case of without PT, the late decay of $\chi$ contributions dominates the DM relic density of $N_{1}$, and the much smaller $f_{1}$ and $h_{1}$ are chosen here to make sure the correct relic density $\Omega h^{2}=0.12$ [38] can be obtained. The magnitude of $h_{1}$ is relatively larger than $f_{1}$ to ensure the $\chi$ particle can fully decay before $\mathrm{BBN}$.

\section{GRAVITATIONAL WAVES FROM THE SFOEWPT}

The gravitational wave (GW) signals produced during the phase transition process can be characterized by two parameters of $\alpha$ and $\beta$ at the phase transition temperature $T_{\star}$ (bubble nucleation temperature). The first important parameter $\alpha$ is the latent heat normalized by the radiation energy, given by $\alpha=\epsilon / \rho_{\text {rad }}$ with $\rho_{\text {rad }}=\pi^{2} g_{\star} T_{*}^{4} / 30$. The latent heat $\epsilon$ includes the difference of the vacuum energy between the false and true vacuum and the entropy variation $\Delta s$ (see Appendix D) at the phase transition temperature, given by

$$
\epsilon=-\Delta V-T \Delta s=\left.\left(-\Delta V+T \frac{\partial V}{\partial T}\right)\right|_{T=T_{\star}} .
$$

The second crucial parameter $\beta$ reflects the duration of the phase transition and characterizes the peak frequency of the GW spectrum. Under the assumptions of adiabatic expansion of the Universe, one has

$$
\frac{\beta}{H_{\star}}=\left.T_{\star} \frac{d}{d T}\left(\frac{S_{3}}{T}\right)\right|_{T_{\star}},
$$

where $S_{3}$ is the three-dimensional Euclidean action for the critical bubble (nucleating bubble).

With the two parameters at hand, one can calculate the gravitational wave signals produced by the SFOEWPT, which includes three dominant contributions: bubble collisions, sound waves, and magnetohydrodynamic turbulence (MHD) in the plasma [52,53]. The total energy spectrum is given by

$$
\Omega_{\mathrm{GW}} h^{2} \simeq \Omega_{\mathrm{col}} h^{2}+\Omega_{\mathrm{sw}} h^{2}+\Omega_{\mathrm{turb}} h^{2} .
$$

The first important contribution is the bubble collision, estimating with the envelope approximation [54-56], which is given by [57]

$$
\begin{aligned}
\Omega_{\mathrm{col}} h^{2}= & 1.67 \times 10^{-5}\left(\frac{H_{*}}{\beta}\right)^{2}\left(\frac{\kappa \alpha}{1+\alpha}\right)^{2}\left(\frac{100}{g_{*}}\right)^{1 / 3} \\
& \times\left(\frac{0.11 v_{b}^{3}}{0.42+v_{b}^{2}}\right) \frac{3.8\left(f / f_{\text {env }}\right)^{2.8}}{1+2.8\left(f / f_{\text {env }}\right)^{3.8}},
\end{aligned}
$$

with the bubble wall velocity $v_{b}$ and the efficient factor $\kappa$ being functions of the crucial parameter $\alpha$ [58],

$v_{b} \simeq \frac{1 / \sqrt{3}+\sqrt{\alpha^{2}+2 \alpha / 3}}{1+\alpha}, \quad \kappa \simeq \frac{0.715 \alpha+\frac{4}{27} \sqrt{3 \alpha / 2}}{1+0.715 \alpha}$,

and the peak frequency located at

$f_{\text {env }}=16.5 \times 10^{-6}\left(\frac{f_{*}}{H_{*}}\right)\left(\frac{T_{*}}{100 \mathrm{GeV}}\right)\left(\frac{g_{*}}{100}\right)^{1 / 6} \mathrm{~Hz}$.

The other two main contributions are the sound waves and the MHD, which are given, respectively, by

$$
\begin{aligned}
\Omega_{\mathrm{sw}} h^{2}= & 2.65 \times 10^{-6}\left(\frac{H_{*}}{\beta}\right)\left(\frac{\kappa_{v} \alpha}{1+\alpha}\right)^{2}\left(\frac{100}{g_{*}}\right)^{1 / 3} v_{b}\left(\frac{f}{f_{\mathrm{sw}}}\right)^{3} \\
& \times\left(\frac{7}{4+3\left(f / f_{\mathrm{sw}}\right)^{2}}\right)^{7 / 2} \\
\Omega_{\mathrm{turb}} h^{2}= & 3.35 \times 10^{-4}\left(\frac{H_{*}}{\beta}\right)\left(\frac{\kappa_{\mathrm{turb}} \alpha}{1+\alpha}\right)^{3 / 2}\left(\frac{100}{g_{*}}\right)^{1 / 3} v_{b} \\
& \times \frac{\left(f / f_{\text {turb }}\right)^{3}}{\left[1+\left(f / f_{\text {turb }}\right)\right]^{11 / 3}\left(1+8 \pi f / h_{*}\right)}
\end{aligned}
$$

with $\kappa_{v} \approx \alpha(0.73+0.083 \sqrt{\alpha}+\alpha)^{-1} \quad$ and $\quad \kappa_{\text {turb }} \approx 0.1 \kappa_{v}$ describing the fraction of latent heat transformed into the

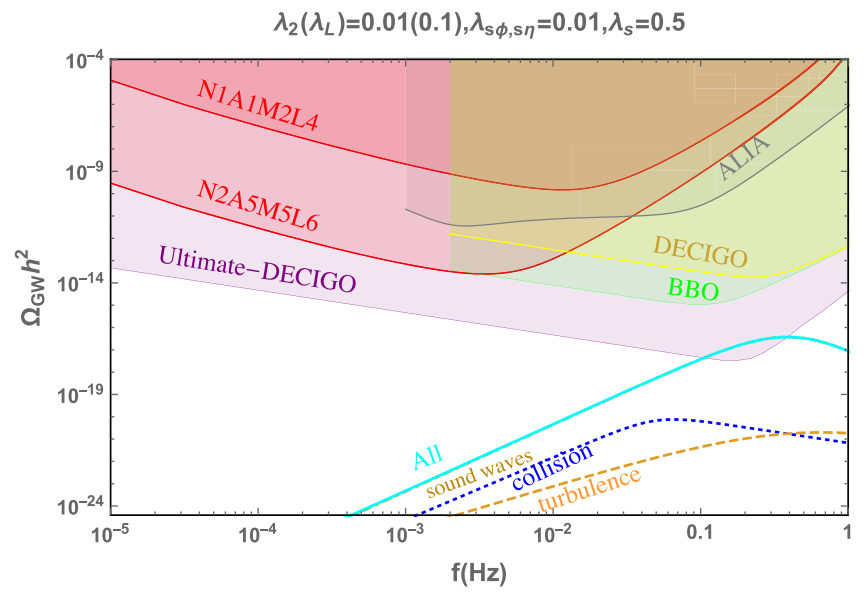

FIG. 11. The GW signal spectrum for the phase transition pattern of $O \rightarrow C$ with $m_{A, H^{ \pm}}=450 \mathrm{GeV}$ and $m_{H}\left(m_{\chi}\right)=$ $64(100) \mathrm{GeV}$, and the mixing angle is $\theta=0.01$. 

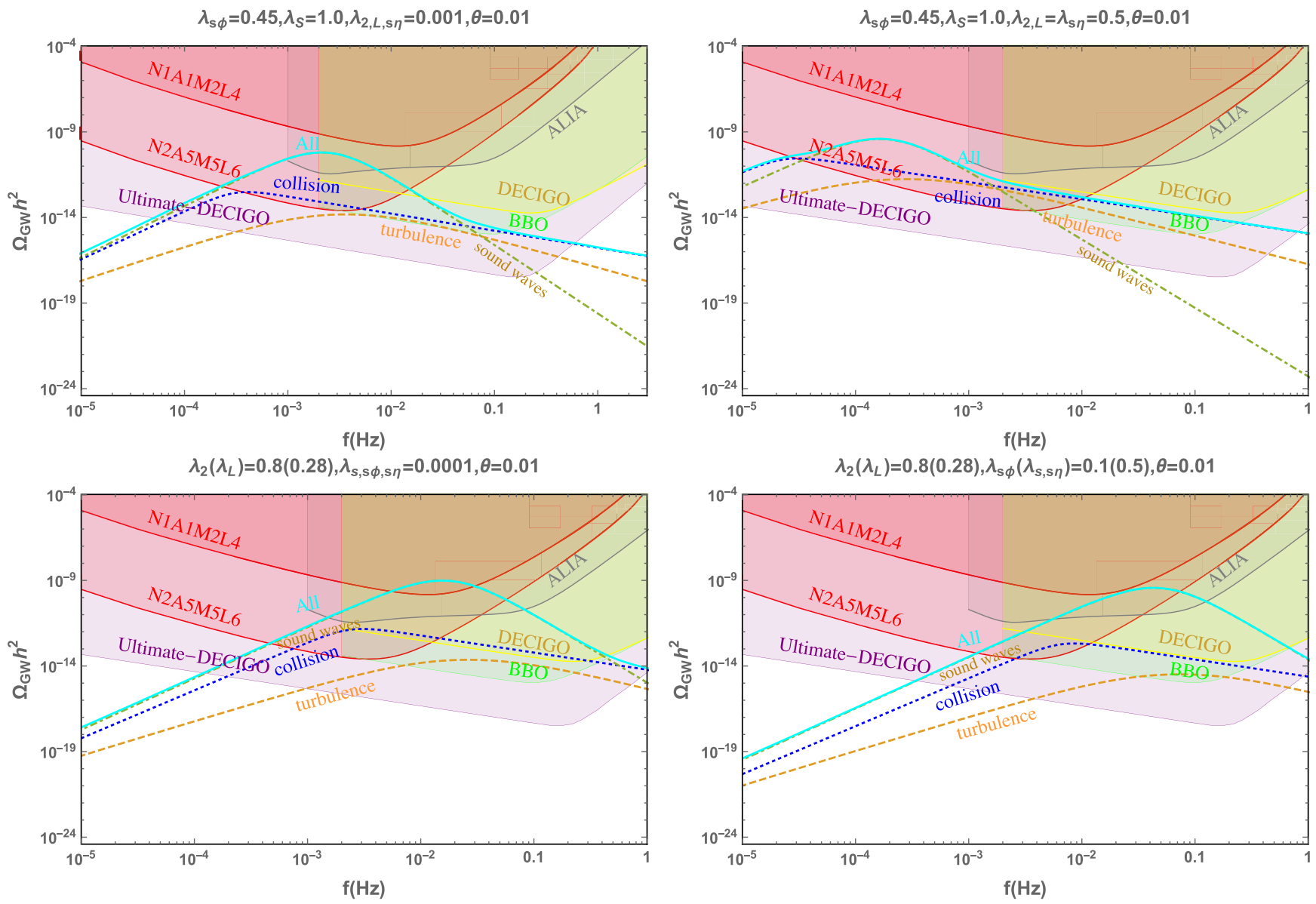

FIG. 12. The GW signal spectrum for the phase transition patterns of $O \rightarrow A \rightarrow C(O \rightarrow B \rightarrow C)$ for up (down) plots with the scalar masses $m_{\chi}=100 \mathrm{GeV}$ and $m_{A, H, H^{ \pm}}=300 \mathrm{GeV}\left[m_{A, H^{ \pm}}=300 \mathrm{GeV}\right.$ and $\left.m_{H}\left(m_{\chi}\right)=85(100) \mathrm{GeV}\right]$.

bulk motion of the fluid for sound waves and MHD. Today's Hubble parameter is

$$
h_{*}=1.65 \times 10^{-2} \mathrm{~m} \mathrm{~Hz} \frac{T_{*}}{100 \mathrm{GeV}}\left(\frac{g_{*}}{100}\right)^{1 / 6} .
$$

The peak frequencies of sound waves and MHD locate at

$$
f_{\mathrm{sw}}=1.9 \times 10^{-5} \frac{1}{v_{b}}\left(\frac{\beta}{H_{*}}\right)\left(\frac{T_{*}}{100 \mathrm{GeV}}\right)\left(\frac{g_{*}}{100}\right)^{1 / 6} \mathrm{~Hz},
$$

$$
f_{\text {turb }}=2.7 \times 10^{-5} \frac{1}{v_{b}}\left(\frac{\beta}{H_{*}}\right)\left(\frac{T_{*}}{100 \mathrm{GeV}}\right)\left(\frac{g_{*}}{100}\right)^{1 / 6} \mathrm{~Hz} .
$$

For the one-step SFOEWPT pattern $O \rightarrow C$, one can consider the energy barrier for the phase transition as almost coming from the singlet part or the inert doublet part, since the mixing of the two scalars is assumed to be negligible. For the inert doublet dominant case, we refer to Fig. 11, which is an analogy to the study of Ref. [45], while the singlet dominant case requires large Higgs portal coupling $\lambda_{s \phi}$; see Ref. [33]. In Fig. 12, we plot the GW signals to be probed by the ALIA, eLISA, BBO, DECIGO, and ultimate-DECIGO experiments, which are generated from the two-step SFOEWPT with patterns $O \rightarrow A \rightarrow C$ and $O \rightarrow B \rightarrow C$. The top plots in Fig. 12 indicate that the increase of $\lambda_{2, L, s \eta}$ leads to the shift of the frequency to a smaller value. The parameter $\lambda_{2, L, s \eta}$ chosen for the top-right panel ensures that the inert scalars and the singlet scalar live in the thermal bath, which is the benchmark model corresponding to the freeze-in production of $N_{1}$ with the $O \rightarrow A \rightarrow C$ scenario, as studied in the left panel in Fig. 8. The related studies on GWs in this scenario can be also found in Refs. [59-63]. ${ }^{7}$ The bottom plots show that the peak of the spectrum or frequency shifts to the right by increasing $\lambda_{s, s \eta, s \phi}$ for the $O \rightarrow B \rightarrow C$ pattern. The freezein production of $N_{1}$ without a late decay of $\chi$ (the right panel in Fig. 8) could be obtained with the parameter setup shown in the bottom-right panel in Fig. 12, except

\footnotetext{
${ }^{7}$ For the GW signals from first-order phase transitions from a neutrino mass and dark matter model, see Refs. [64,65].
} 
employing a negligible small $\theta$ that will not affect the GW signal production. Considering the freeze-in production of $\chi$, as studied previously in Sec. IV B (see Fig. 10), the negligibly small $\lambda_{h s, s \phi}$ and $\theta$ are required which also do not affect the phase transition dynamics. This can be realized due to the pattern $O \rightarrow B \rightarrow C$ being the inert doubletlike two-step phase transition. The study of the GWs from the inert doublet two-step phase transition is absent in the literature, and we fill the blank in this scenario.

\section{COMMENTS ON COLLIDER SEARCHES}

One of the signals predicted by the SFOEWPT is the deviation of the triple Higgs couplings, which might be able to be probed at colliders, such as the lepton colliders $[66,67]$ and high-energy hadronic colliders [30]. We emphasize that this kind of search mostly applies to the SFOEWPT through the one-step pattern with sizable dimensional-six operators after heavy particles are integrated out. For the phase transition patterns $O \rightarrow A \rightarrow C$ and $O \rightarrow B \rightarrow C$, no sizable dimensional-six operators could be obtained, since there is no contribution from the tree level. In both cases, the dimensional-six operators can be obtained at the one-loop level; see Refs. [44,68] for the $O \rightarrow A \rightarrow C$ pattern and Ref. [45] for the $O \rightarrow B \rightarrow C$ pattern. As argued in Ref. [28], both scenarios might be tested at the LHC with the off-shell $Z$-pair channel [69] and at the future lepton colliders $[40,44,63]$ as well as a $100 \mathrm{TeV} p p$ collider [33]. As studied in Ref. [40], the displaced vertex signature might be able to tell the difference between the scalar sectors of this model and the inert doublet model. We checked the charged Higgs contribution to the Higgs diphoton signal following Ref. [40], and the deviation from the SM prediction is negligible for the FIMP benchmark scenarios under study.

For the FIMP DM $N_{1}$, the decay lengths of $\chi, H, A, H^{ \pm}$ particles are far beyond the ability of the LHC. For the scenario of $m_{N_{3}}>m_{H^{ \pm}, A, H, \chi}>m_{N_{2}}>m_{N_{1}}$, as studied in Ref. [41], one can expect, for the relic abundance of $N_{1}$ DM coming from the late decay of $N_{2}$ after the freeze-out and before the BBN epoch, the decays of the heavier singlet fermions $N_{2} \rightarrow N_{1} \bar{\ell} \ell$ mediated by the scalars (ignore the mixing of $H$ and $\chi$ ) should happen with $H\left(T=m_{N_{2}} / 20\right)>$ $\Gamma_{1}\left(N_{2} \rightarrow N_{1} \bar{\ell}_{\alpha} \ell_{\beta}\right)>H(T=1 \mathrm{MeV})$ and

$$
\begin{aligned}
\Gamma_{1}\left(N_{2,3} \rightarrow N_{1} \bar{\ell}_{\alpha} \ell_{\beta}\right)= & \frac{m_{N_{2,3}}^{5}}{6144 \pi^{3} m_{H^{ \pm}, H, A}^{4}} \\
& \times\left(\left|f_{\alpha 1}\right|^{2}\left|f_{\beta 2,3}\right|^{2}+\left|f_{\beta 1}\right|^{2}\left|f_{\alpha 2,3}\right|^{2}\right), \\
\Gamma_{2}\left(N_{2,3} \rightarrow N_{1} \bar{\ell}_{\alpha} \ell_{\beta}\right)= & \frac{m_{N_{2,3}}^{5}}{6144 \pi^{3} m_{\chi}^{4}} \\
& \times\left(\left|h_{\alpha 1}\right|^{2}\left|h_{\beta 2,3}\right|^{2}+\left|h_{\beta 1}\right|^{2}\left|h_{\alpha 2,3}\right|^{2}\right) .
\end{aligned}
$$

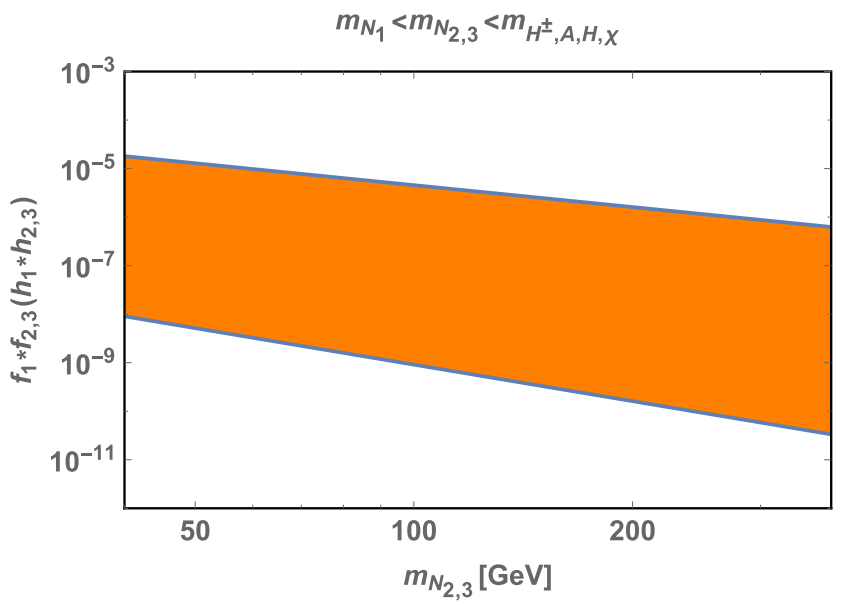

FIG. 13. BBN allowed parameter spaces of $m_{N_{2,3}}$ and $f_{1}^{2} f_{2,3}^{2} \times$ $\left(h_{1}^{2} h_{2,3}^{2}\right)$.

Therefore, we obtain parameter regions allowed by BBN, which is shown in Fig. 13. The freeze-in mechanism of DM $N_{1}$ production is not valid in this scenario due to the largess of the Yukawa couplings.

Reference [46] studied the LHC dilepton pair signal in the FIMP DM scenario of the scotogenic model proposed by Ma [42], which can be applied to our study except the late decay, which calls for much heavier $m_{N_{23}}$; see Fig. 9. In the model under study, the dilepton decay for $m_{H^{ \pm}, A, H, \chi}>$ $m_{N_{3}}>m_{N_{2}}>m_{N_{1}}$ is given by

$$
\begin{aligned}
\Gamma_{1}^{\prime}\left(N_{3} \rightarrow N_{2} \bar{\ell}_{\alpha} \ell_{\beta}\right)= & \frac{m_{N_{3}}^{5}}{6144 \pi^{3} m_{H^{ \pm}, H, A}^{4}} \\
& \times\left(\left|f_{\alpha 2}\right|^{2}\left|f_{\beta 3}\right|^{2}+\left|f_{\beta 3}\right|^{2}\left|f_{\alpha 2}\right|^{2}\right),
\end{aligned}
$$

$\Gamma_{2}^{\prime}\left(N_{3} \rightarrow N_{2} \bar{\ell}_{\alpha} \ell_{\beta}\right)=\frac{m_{N_{3}}^{5}}{6144 \pi^{3} m_{\chi}^{4}}$

$$
\times\left(\left|h_{\alpha 2}\right|^{2}\left|h_{\beta 3}\right|^{2}+\left|h_{\beta 3}\right|^{2}\left|h_{\alpha 2}\right|^{2}\right),
$$

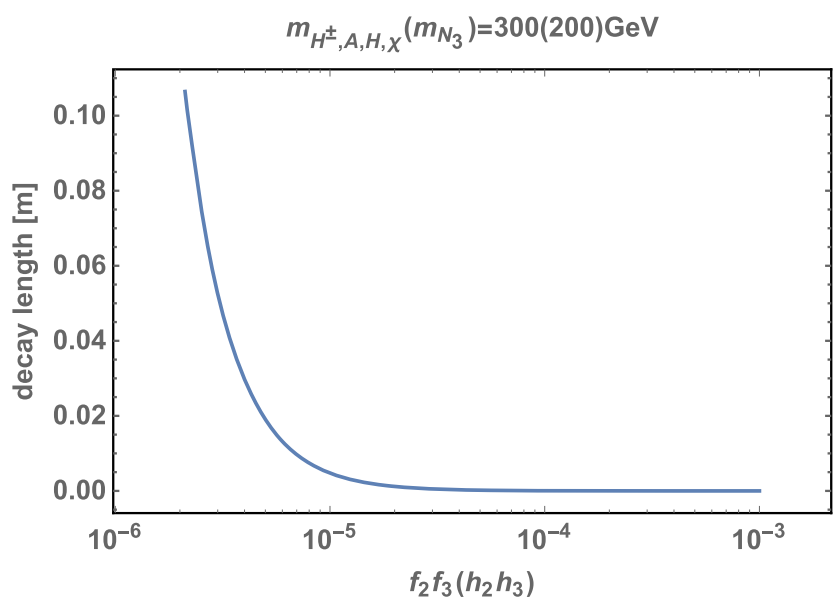

FIG. 14. The decay length of $N_{3}$ as a function of $h_{2 \alpha} h_{3 \beta} \times$ $\left(f_{2 \alpha} f_{3 \beta}\right)$. 
where we have an additional decay channel of $N_{3} \rightarrow N_{2} \bar{\ell}_{\alpha} \ell_{\beta}$. The decay length is shown in Fig. 14, which means the channel can be tested at the LHC.

\section{CONCLUSION AND DISCUSSION}

This work demonstrates that the two-step SFOEWPT modifies the thermal history of the FIMP DM production through modifying the kinematical thresholds of the decay and scattering processes. The phase transition effects may result in a larger or smaller DM relic density in comparison with the traditional calculation. We study (i) the FIMP DM production via the $2 \rightarrow 2$ scattering processes and/or the decay of the particles from the thermal bath; and (ii) the DM production with the late decay of a neutral scalar, which is also produced through the freeze-in mechanism. The hidden scalars that induce the two-step SFOEWPT would lead to detectable signals at colliders and the gravitational wave signals to be probed on the future space-based interferometer. The realization of the second scenario here requires a tiny mixing angle between the inert $C P$-even neutral scalar and the singlet scalar to accommodate massive neutrinos of the scotogenic model. The SFOEWPT modified FIMP DM is general and applies to the context of DM models with more than one hidden sector.

For the explanation of the baryon asymmetry of the Universe utilizing the electroweak baryogenesis mechanism [70], extra $C P$-violation operators involving the dark sector are necessary $[61,62,71,72]$. For the leptogenesis realization in the scotogenic model and its connection with the WIMP DM, we refer to Refs. [73-76].

\section{ACKNOWLEDGMENTS}

We are grateful to Lian-Tao Wang, Stefano Profumo, and Michael A. Schmidt for communication on the entropy during the EWPT process. We thank Oscar Zapata, Probir Roy, and Takashi Toma for discussions on the nonthermal dark matter production mechanisms; Huai-Ke Guo for helpful communications on the GWs from the SFOEWPT; and Zhi-Long Han for helpful discussions on the scotogenic models. The work of L. B. is supported by the National Natural Science Foundation of China (under Grants No. 11605016 and No. 11647307), Basic Science Research Program through the National Research Foundation of Korea (NRF) funded by the Ministry of Education, Science and Technology (NRF2016R1A2B4008759), and Korea Research Fellowship Program through the National Research Foundation of Korea (NRF) funded by the Ministry of Science and ICT (2017H1D3A1A01014046). The work of X. L. is supported in part by the National Postdoctoral Program for Innovative Talents under Grant No. BX201700116, by the Jiangsu Planned Projects for Postdoctoral Research Funds under Grant No. 1701130B, by the National Natural Science Foundation of China under Grants No. 11775109 and
No. U1738134, and by the Nanjing University Grant No. 14902303.

\section{APPENDIX A: THEORETICAL AND EXPERIMENTAL CONSTRAINTS}

\section{Parameter relations}

In the basis of real neutral scalars, $\left(S, H_{0}, A\right)$, the mass matrix for the dark scalars is

$$
\mathbf{M}^{2}=\left(\begin{array}{ccc}
2 \mu_{S}^{2}+v^{2} \lambda_{s \phi} & v \mu_{\text {soft }} & 0 \\
v \mu_{\text {soft }} & \mu_{\eta}^{2}+v^{2} \lambda_{L} & 0 \\
0 & 0 & \mu_{\eta}^{2}+v^{2} \lambda_{S}
\end{array}\right) .
$$

The masses and interactions of the scalar sector are parametrized by the scalar quartic couplings and $\mu_{\Phi, \eta, S \text {,soft }}$, which are in terms of the physical masses in the global EW vacuum:

$$
\begin{aligned}
\lambda_{3} & =\frac{2}{v^{2}}\left(-m_{H}^{2} \cos ^{2} \theta+m_{H^{ \pm}}^{2}-m_{\chi}^{2} \sin ^{2} \theta+\lambda_{L} v^{2}\right), \\
\lambda_{4} & =\frac{1}{v^{2}}\left(m_{A}^{2}+m_{H}^{2} \cos ^{2} \theta-2 m_{H^{ \pm}}^{2}+m_{\chi}^{2} \sin ^{2} \theta\right), \\
\lambda_{5} & =\frac{1}{v^{2}}\left(-m_{A}^{2}+m_{H}^{2} \cos ^{2} \theta+m_{\chi}^{2} \sin ^{2} \theta\right), \\
\mu_{S}^{2} & =\frac{1}{2}\left(m_{\chi}^{2} \cos ^{2} \theta+m_{H}^{2} \sin ^{2} \theta-\lambda_{s \phi} v^{2}\right), \\
\mu_{\eta}^{2} & =m_{H}^{2} \cos ^{2} \theta+m_{\chi}^{2} \sin ^{2} \theta-\lambda_{L} v^{2} \\
\mu_{\mathrm{soft}} & =\frac{1}{2 v}\left(m_{\chi}^{2}-m_{H}^{2}\right) \sin 2 \theta
\end{aligned}
$$

with $\lambda_{L, S}=\left(\lambda_{3}+\lambda_{4} \pm \lambda_{5}\right) / 2$.

\section{Perturbative unitarity}

All the scattering matrices of the model are [40]

$$
\begin{aligned}
8 \pi S_{Y=2, \sigma=1} & =\left(\begin{array}{ccc}
2 \lambda_{1} & \lambda_{5} & 0 \\
\lambda_{5}^{*} & 2 \lambda_{2} & 0 \\
0 & 0 & \lambda_{3}+\lambda_{4}
\end{array}\right), \\
8 \pi S_{Y=2, \sigma=0}=\lambda_{3}-\lambda_{4}, & \\
8 \pi S_{Y=0, \sigma=1} & =\left(\begin{array}{cccc}
2 \lambda_{1} & \lambda_{4} & 0 & 0 \\
\lambda_{4} & 2 \lambda_{2} & 0 & 0 \\
0 & 0 & \lambda_{3} & \lambda_{5}^{*} \\
0 & 0 & \lambda_{5} & \lambda_{3}
\end{array}\right), \\
8 \pi S_{Y=1, \sigma=1 / 2} & =\left(\begin{array}{ccc}
2 \lambda_{s \phi} & 0 \\
0 & 2 \lambda_{s \eta}
\end{array}\right),
\end{aligned}
$$




$$
8 \pi S_{Y=0, \sigma=0}=\left(\begin{array}{ccccc}
6 \lambda_{1} & 2 \lambda_{3}+\lambda_{4} & \sqrt{2} \lambda_{s \phi} & 0 & 0 \\
2 \lambda_{3}+\lambda_{4} & 6 \lambda_{2} & \sqrt{2} \lambda_{s \eta} & 0 & 0 \\
\sqrt{2} \lambda_{s \phi} & \sqrt{2} \lambda_{s \eta} & \lambda_{S} / 4 & 0 & 0 \\
0 & 0 & 0 & \lambda_{3}+2 \lambda_{4} & 3 \lambda_{5}^{*} \\
0 & 0 & 0 & 3 \lambda_{5} & \lambda_{3}+2 \lambda_{4}
\end{array}\right) .
$$

Then the eigenvalues $\Lambda_{Y \sigma i}^{X}$ of the above scattering matrices (where $i= \pm$, or 1,2,3) can be calculated as

$$
\begin{gathered}
\Lambda_{21 \pm}^{\text {even }}=\lambda_{1}+\lambda_{2} \pm \sqrt{\left(\lambda_{1}-\lambda_{2}\right)^{2}+\left|\lambda_{5}\right|^{2}}, \quad \Lambda_{21}^{\text {odd }}=\lambda_{3}+\lambda_{4}, \quad \Lambda_{20}^{\text {odd }}=\lambda_{3}-\lambda_{4} \\
\Lambda_{01 \pm}^{\text {even }}=\lambda_{1}+\lambda_{2} \pm \sqrt{\left(\lambda_{1}-\lambda_{2}\right)^{2}+\lambda_{4}^{2}}, \quad \Lambda_{01 \pm}^{\text {odd }}=\lambda_{3} \pm\left|\lambda_{5}\right| \\
\Lambda_{00 \pm}^{\text {odd }}=\lambda_{3}+2 \lambda_{4} \pm 3\left|\lambda_{5}\right|,
\end{gathered}
$$

and the $\Lambda_{001,2,3}^{\text {even }}$ correspond to the three roots of the polynomial equation

$$
\begin{aligned}
x^{3} & -x^{2}\left(6 \lambda_{1}+6 \lambda_{2}+\lambda_{s} / 4\right)+x\left(36 \lambda_{1} \lambda_{2}-4 \lambda_{3}^{2}-4 \lambda_{3} \lambda_{4}-\lambda_{4}^{2}+3 \lambda_{1} \lambda_{s} / 2+3 \lambda_{2} \lambda_{s} / 2-2 \lambda_{s \phi}^{2}-2 \lambda_{s \eta}^{2}\right)+12 \lambda_{1} \lambda_{s \eta}^{2}+12 \lambda_{2} \lambda_{s \phi}^{2} \\
& -4 \lambda_{4} \lambda_{s \phi} \lambda_{s \eta}-8 \lambda_{3} \lambda_{s \phi} \lambda_{s \eta}+\lambda_{4}^{2} \lambda_{s} / 4+\lambda_{3} \lambda_{4} \lambda_{s}+\lambda_{3}^{2} \lambda_{s}-9 \lambda_{1} \lambda_{2} \lambda_{s}=0 .
\end{aligned}
$$

\section{Vacuum stability conditions}

The vacuum stability conditions for the model are given as [40]

$$
\lambda_{1}, \lambda_{2}, \lambda_{s}>0, \quad \sqrt{\lambda_{1} \lambda_{s}}+\lambda_{s \phi}>0, \quad \sqrt{\lambda_{2} \lambda_{s}}+\lambda_{s \eta}>0
$$

and, for the $\lambda_{4}>0$ case,

$$
\begin{gathered}
2 \sqrt{\lambda_{1} \lambda_{2}}+\lambda_{3}>0, \\
\sqrt{\lambda_{1} \lambda_{2} \lambda_{s}} / 2+\sqrt{\lambda_{1}} \lambda_{s \eta}+\sqrt{\lambda_{2}} \lambda_{s \phi}+\sqrt{\lambda_{s}} \lambda_{3} / 2+\sqrt{\left(\sqrt{\lambda_{1} \lambda_{s}}+\lambda_{s \phi}\right)\left(2 \sqrt{\lambda_{2} \lambda_{s \phi}}+\lambda_{s \eta}\right)\left(2 \sqrt{\lambda_{1} \lambda_{2}}+\lambda_{3}\right)}>0
\end{gathered}
$$

if $\lambda_{4}-\left|\lambda_{5}\right|<0$, one obtains [77]

$$
\begin{gathered}
2 \sqrt{\lambda_{1} \lambda_{2}}+\lambda_{3}+\lambda_{4}-\left|\lambda_{5}\right|>0, \\
\sqrt{\lambda_{1} \lambda_{2} \lambda_{s}} / 2+\sqrt{\lambda_{1}} \lambda_{s \eta}+\sqrt{\lambda_{2}} \lambda_{s \phi}+\sqrt{\lambda_{s}}\left(\lambda_{3}+\lambda_{4}-\left|\lambda_{5}\right|\right) / 2+\sqrt{\left(\sqrt{\lambda_{1} \lambda_{s}}+\lambda_{s \phi}\right)\left(2 \sqrt{\lambda_{2} \lambda_{s}}+\lambda_{s \eta}\right)\left(2 \sqrt{\lambda_{1} \lambda_{2}}+\lambda_{3}+\lambda_{4}-\left|\lambda_{5}\right|\right)}>0 .
\end{gathered}
$$

\section{The $T$ parameter}

The $T$ parameter is given by $T=\Delta \rho / \alpha_{\mathrm{EM}}$ [78], where [40]

$$
\begin{aligned}
\Delta \rho= & \frac{\left(\lambda_{4}+\lambda_{5}\right)^{2}}{2}\left(\cos ^{2} \theta f\left(m_{H^{ \pm}}, m_{H}\right)+\sin ^{2} \theta f\left(m_{H^{ \pm}}, m_{\chi}\right)\right)+\frac{\left(\lambda_{4}-\lambda_{5}\right)^{2}}{2} f\left(m_{H^{ \pm}}, m_{A}\right)-2\left(\lambda_{5} \cos \theta-\mu_{\mathrm{soft}} \sin \theta / v\right)^{2} f\left(m_{A}, m_{H}\right) \\
& -2\left(\lambda_{5} \sin \theta+\mu_{\mathrm{soft}} \cos \theta / v\right)^{2} f\left(m_{A}, m_{\chi}\right), \\
\text { with } f\left(m_{1}, m_{2}\right) & =\frac{v^{2}}{32 \pi^{2}} \int_{0}^{1} \frac{d x x(1-x)}{x m_{1}^{2}+(1-x) m_{2}^{2}},
\end{aligned}
$$

where $\mu_{\text {soft }}$ and $\lambda_{4,5}$ are given in Eq. (A7). 


\section{LEP bounds}

Considering the $C P$-even neutral Higgs $H$ is a mixture of doublet and singlet, the LEP bounds on the scalar masses of this model are the same as in inert doublet model. The lower limits on the scalar masses come from the precise measurements of the $W$ and $Z$ widths:

$$
\begin{aligned}
m_{H}+m_{H^{ \pm}}>m_{W^{ \pm}}, & m_{A}+m_{H^{ \pm}}>m_{W^{ \pm}}, \\
m_{H}+m_{A}>m_{Z}, & 2 m_{H^{ \pm}}>m_{Z} .
\end{aligned}
$$

Utilizing the neutralino search at LEP II, the mass splitting $\Delta m_{H A}$ should be either smaller than $8 \mathrm{GeV}$ or greater than $100 \mathrm{GeV}$ for $m_{A}<80 \mathrm{GeV}$ [79]. The production of the charged Higgs pairs $H^{+} H^{-}$at LEP II give rise to [80]

$$
m_{H^{ \pm}}>70 \mathrm{GeV}
$$

\section{On the massive neutrino mass and the constraints on the model parameters}

For a large mass splitting of $m_{\chi}$ and $m_{H}$ and large $m_{N_{2,3}}$, Eq. (2.6) reduces to

$$
\begin{aligned}
\left(M_{\nu}\right)_{\alpha \beta}= & \frac{\sin 2 \theta}{32 \sqrt{2} \pi^{2}} \sum_{\kappa=2,3} f_{\alpha \kappa} h_{\kappa \beta} m_{N_{\kappa}} \\
& \times\left[\frac{m_{H}^{2}}{m_{N_{\kappa}}^{2}} \log \frac{m_{H}^{2}}{m_{N_{\kappa}}^{2}}-\frac{m_{\chi}^{2}}{m_{N_{\kappa}}^{2}} \log \frac{m_{\chi}^{2}}{m_{N_{\kappa}}^{2}}\right] .
\end{aligned}
$$

The corresponding parameter limits from the neutrino mass are plotted in Fig. 15, and a larger mixing angle $\theta$ is preferred for a lower magnitude of $f_{\alpha 2,3} h_{2,3 \beta}$.

Considering the $N_{1}$ decoupled from the neutrino mass matrix, when $m_{\chi} \sim m_{H}$ the neutrino mass matrix of Eq. (2.6) reduces to

$$
\begin{aligned}
\left(M_{\nu}\right)_{\alpha \beta}= & \frac{\sin 2 \theta}{32 \sqrt{2} \pi^{2}} \sum_{\kappa=2,3} f_{\alpha \kappa} h_{\kappa \beta} \frac{m_{N_{\kappa}}\left(m_{H}^{2}-m_{\chi}^{2}\right)}{m_{H \chi}^{2}-m_{N_{\kappa}}^{2}} \\
& \times\left(1-\frac{m_{N_{\kappa}}^{2}}{m_{H \chi}^{2}-m_{N_{\kappa}}^{2}} \log \left(\frac{m_{H_{\chi}}^{2}}{m_{N_{\kappa}}^{2}}\right)\right),
\end{aligned}
$$

with $m_{H \chi}^{2}=\left(m_{H}^{2}+m_{\chi}^{2}\right) / 2$. With Eq. (A7), for the parameter set of $m_{H \chi}^{2} \ll m_{N_{\kappa}}^{2}$, Eq. (A25) casts the form of

$$
\begin{aligned}
\left(M_{\nu}\right)_{\alpha \beta} & =\frac{\sin 2 \theta}{32 \sqrt{2} \pi^{2}} \sum_{\kappa=2,3} f_{\alpha \kappa} h_{\kappa \beta} \frac{\left(m_{H}^{2}-m_{\chi}^{2}\right)}{m_{N_{\kappa}}}\left(\log \frac{m_{N_{\kappa}}^{2}}{m_{H \chi}^{2}}-1\right) \\
& =\frac{1}{32 \sqrt{2} \pi^{2}} \sum_{\kappa} f_{\alpha \kappa} h_{\kappa \beta} \frac{-2 v \mu_{\mathrm{soft}}}{m_{N_{\kappa}}}\left(\log \frac{m_{N_{\kappa}}^{2}}{m_{H_{\chi}}^{2}}-1\right),
\end{aligned}
$$

whereas, when $m_{H_{\chi}}^{2} \gg m_{N_{\kappa}}^{2}$, Eq. (A25) recasts the form of

$$
\begin{gathered}
\left(M_{\nu}\right)_{\alpha \beta}=\frac{\sin 2 \theta}{32 \sqrt{2} \pi^{2}} \sum_{\kappa=2,3} f_{\alpha \kappa} h_{\kappa \beta} \frac{\left(m_{H}^{2}-m_{\chi}^{2}\right) m_{N_{\kappa}}}{m_{H \chi}^{2}} \\
=\frac{-2 v \mu_{\text {soft }}}{32 \sqrt{2} \pi^{2}} \sum_{\kappa} f_{\alpha \kappa} h_{\kappa \beta} \frac{m_{N_{\kappa}}}{m_{H \chi}^{2}} .
\end{gathered}
$$

Since we assume the $N_{1}$ never reaches equilibrium with the thermal bath, the requirement of the neutrino mass of the order of $\sim 0.1 \mathrm{eV}$ leads to a ballpark estimate of the size of the multiplication of Yukawa couplings $f_{\alpha 2,3} h_{2,3 \beta}$ and the soft terms $\mu_{\text {soft }}$.

\section{APPENDIX B: THERMAL FIELD-DEPENDENT MASSES}

The field-dependent mass of $C P$-even, $C P$-odd, and charged scalars are given as follows:
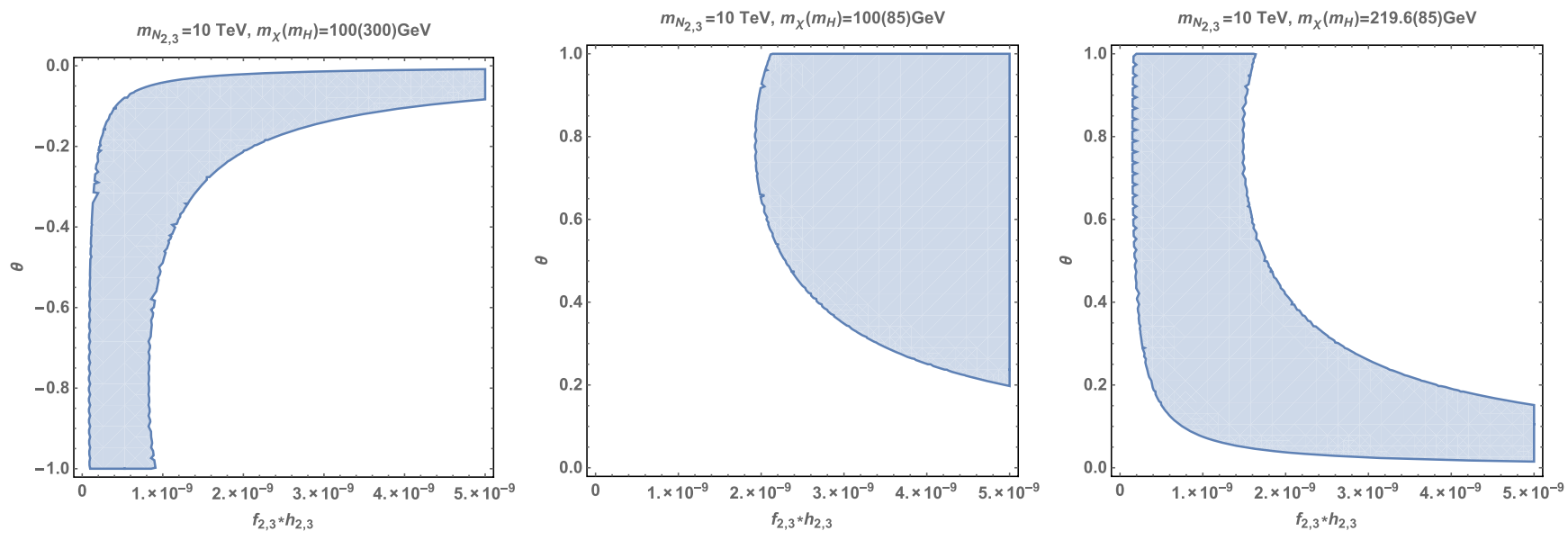

FIG. 15. The neutrino mass bounds on mixing angle $\theta$ and the multiplication of Yukawa $f$ and $h$ for OA (left), OBC (middle), and $\mathrm{OBC}$ with a late decay (right). 


$$
\begin{aligned}
M_{h}^{2} & =\left(\begin{array}{ccc}
3 \lambda_{1} h^{2}+\lambda_{L} H_{0}^{2}+\lambda_{s \phi} S^{2}+\mu_{\phi}^{2} & 2 \lambda_{L} h H_{0}+\mu_{\mathrm{soft}} S & 2 \lambda_{s \phi} h S+\mu_{\mathrm{soft}} H_{0} \\
2 \lambda_{L} h H_{0}+\mu_{\mathrm{soft}} S & \lambda_{L} h^{2}+3 \lambda_{2} H_{0}^{2}+\lambda_{s \eta} S^{2}+\mu_{\eta}^{2} & 2 \lambda_{s \eta} H_{0} S+\mu_{\text {soft }} h \\
2 \lambda_{s \phi} h S+\mu_{\mathrm{soft}} H_{0} & 2 \lambda_{s \eta} H_{0} S+\mu_{\mathrm{soft}} h & \lambda_{s \phi} h^{2}+3 \lambda_{s} S^{2}+\lambda_{s \eta} H_{0}^{2}+\mu_{s}^{2}
\end{array}\right), \\
M_{A}^{2} & =\left(\begin{array}{cc}
\lambda_{1} h^{2}+\lambda_{L} H_{0}^{2}+\lambda_{s \phi} S^{2}+\mu_{\phi}^{2} & \lambda_{5} h H_{0}+\mu_{\mathrm{soft}} S \\
\lambda_{5} h H_{0}+\mu_{\mathrm{soft}} S & \lambda_{S} h^{2}+\lambda_{2} H_{0}^{2}+\lambda_{s \eta} S^{2}+\mu_{\eta}^{2}
\end{array}\right), \\
M_{H^{ \pm}}^{2} & =\left(\begin{array}{cc}
\lambda_{1} h^{2}+\frac{\lambda_{3}}{2} H_{0}^{2}+\lambda_{s \phi} S^{2}+\mu_{\phi}^{2} & \frac{\lambda_{4}+\lambda_{5}}{2} h H_{0}+\mu_{\mathrm{soft}} S \\
\frac{\lambda_{4}+\lambda_{5}}{2} h H_{0}+\mu_{\mathrm{soft}} S & \frac{\lambda_{3}}{2} h^{2}+\lambda_{2} H_{0}^{2}+\lambda_{s \eta} S^{2}+\mu_{\eta}^{2}
\end{array}\right) .
\end{aligned}
$$

The field-dependent thermal masses of scalar masses can be obtained by the replacement of $\mu_{i}^{2} \rightarrow \mu_{i}^{2}(T)$ given in Eq. (3.10). As the temperature cools down, the thermal masses of the neutral $\left[m_{\chi, H, A}(T)\right]$ and charged Higgs bosons $\left[m_{H^{ \pm}}(T)\right]$ for the study of thermally modified FIMPs is different in the symmetric phase, the $Z_{2}$ broken phase, and the EW symmetry broken phases. In the symmetric phase, we have

$$
\begin{gathered}
M_{h}^{\text {sys }}(T)=\left.M_{h}(T)\right|_{\left\langle h, H_{0}, S\right\rangle \rightarrow 0}, \\
M_{A}^{\text {sys }}(T)=\left.M_{A}(T)\right|_{\left\langle h, H_{0}, S\right\rangle \rightarrow 0}, \\
M_{H^{ \pm}}^{\text {sys }}(T)=\left.M_{H^{ \pm}}\right|_{\left\langle h, H_{0}, S\right\rangle \rightarrow 0} .
\end{gathered}
$$

In the $Z_{2}$ broken phase, we have

$$
\begin{gathered}
\left.M_{h}^{\not 2}(T)=\left.M_{h}(T)\right|_{\langle h\rangle \rightarrow 0,\left\langle H_{0}\right.} \text { or } S\right\rangle \rightarrow v_{H}(T) \text { or } \quad v_{s}(T), \\
\left.M_{A}^{\not 2}(T)=\left.M_{A}(T)\right|_{\langle h\rangle \rightarrow 0,\left\langle H_{0}\right.} \text { or } S\right\rangle \rightarrow v_{H}(T) \text { or } \quad v_{s}(T), \\
\left.M_{H^{ \pm}}^{\not 2}(T)=\left.M_{H^{ \pm}}\right|_{\langle h\rangle \rightarrow 0,\left\langle H_{0}\right.} \quad \text { or } \quad S\right\rangle \rightarrow v_{H}(T) \quad \text { or } \quad v_{s}(T),
\end{gathered}
$$

depending on if the first-stage second-order phase transition is the inert phase or the singlet phase. Finally, in the EW broken phase, we have

$$
\begin{gathered}
M_{h}^{\mathrm{EW}}(T)=\left.M_{h}(T)\right|_{\langle h\rangle \rightarrow v(T),\left\langle H_{0}, S\right\rangle \rightarrow 0}, \\
M_{A}^{\mathrm{EW}}(T)=\left.M_{A}(T)\right|_{\langle h\rangle \rightarrow v(T),\left\langle H_{0}, S\right\rangle \rightarrow 0}, \\
M_{H^{ \pm}}^{\mathrm{EW}}(T)=\left.M_{H^{ \pm}}\right|_{\langle h\rangle \rightarrow v(T),\left\langle H_{0}, S\right\rangle \rightarrow 0} .
\end{gathered}
$$

\section{APPENDIX C: THE DM PRODUCTION PROCESS}

For the phase transition pattern $O \rightarrow A \rightarrow C$, the FIMP DM $N_{1}$ production process is dominated by the decay of $X \rightarrow N \ell / \nu$ with $X=\chi, H, A, H \pm$ living in thermal equilibrium. For the phase transition pattern $O \rightarrow B \rightarrow C$, both the decay $\left(H, A, H^{ \pm} \rightarrow N \ell\right)$ and $2 \rightarrow 2$ scattering processes $\left(\gamma H^{ \pm} \rightarrow \bar{N} \ell^{ \pm}\right)$can contribute to the DM production, with the latter dominating. See Fig. 16 for the Feynman diagram for the DM production process. In this scenario, the late decay of $\chi \rightarrow N_{1} \nu$ after freeze-in of $\chi$ may contribute to the DM abundance of $N_{1}$ dominantly, with the subdominant contribution coming from the $2 \rightarrow 2$ scattering process $\left(\gamma H^{ \pm} \rightarrow \bar{N} \ell^{ \pm}\right)$.

With the amplitude of the scattering process being calculated by CalcHEP [81] after the model being implemented in FeynRules [82],

$$
\begin{aligned}
|\mathcal{M}|^{2}= & 4 \pi a_{\mathrm{EW}} f_{N}^{2}\left(\frac{s\left(2\left(m_{\ell}^{2}+M_{N}^{2}\right)-s\right)-M_{H^{ \pm}}^{4}}{\left(M_{H^{ \pm}}^{2}-s\right)^{2}}+\frac{2 m_{\ell}^{2}\left(m_{\ell}^{2}+m_{N}^{2}-m_{H^{ \pm}}^{2}\right)}{\left(m_{\ell}^{2}-t\right)^{2}}\right. \\
& \left.+\frac{-2 m_{\ell}^{4}+2 m_{\ell}^{2}\left(M_{H^{ \pm}}^{2}-s\right)+M_{H^{ \pm}}^{4}-2 M_{H^{ \pm}}^{2} M_{N}^{2}+2 M_{N}^{4}-2 M_{N}^{2} s+s^{2}}{\left(M_{H^{ \pm}}^{2}-s\right)\left(t-m_{\ell}^{2}\right)}\right),
\end{aligned}
$$

with $t=\left(p_{1}(\gamma)-p_{3}(\ell)\right)^{2}$ and $s=\left(p_{1}(\gamma)+p_{2}\left(H^{ \pm}\right)\right)^{2}$, the differential cross section is given by

$$
d \sigma_{\gamma H^{ \pm} \rightarrow \ell N} / d \Omega=\frac{1}{64 \pi^{2}} \frac{\sqrt{1-4 m_{N}^{2} / s}}{s \sqrt{1-4 m_{H^{ \pm}}^{2} / s}}|\mathcal{M}|^{2} .
$$

With the Mandelstam variable $t$ being

$$
\begin{aligned}
t= & m_{\ell}^{2}-2 p_{1}(\gamma) \cdot p_{3}(\ell) \\
\equiv & m_{\ell}^{2}+\frac{\left(m_{H^{ \pm}}^{2}-s\right)}{2 s} \\
& \times\left(m_{\ell}^{2}-\cos \theta \sqrt{m_{\ell}^{4}-2 m_{\ell}^{2}\left(s+m_{N}^{2}\right)+\left(s-m_{N}^{2}\right)^{2}}\right. \\
& \left.+s-m_{N}^{2}\right),
\end{aligned}
$$




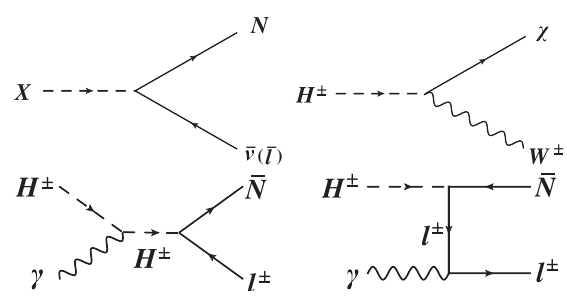

FIG. 16. The DM production process.

one can obtain the Dirac neutrino $N_{1}$ production cross section after integrated out the collide angle $\theta$ :

$$
\sigma_{\gamma H^{ \pm} \rightarrow \ell N}=\frac{1}{32 \pi} \frac{\sqrt{1-4 m_{N}^{2} / s}}{s \sqrt{1-4 m_{H^{ \pm}}^{2} / s}} \int d \cos \theta|\mathcal{M}|^{2} .
$$

\section{APPENDIX D: ENTROPY INJECTED BY EWPT}

In this Appendix, we recall knowledge of entropy deviation induced by EWPT [83]. Assuming that reheating happens quickly relative to the expansion rate, the energy density $\rho$ of the Universe does not change during reheating. And there is only a small amount of reheating by the released latent heat of the transition. We do not expect the phase coexistence stage for SFOEWPT.
The injection of entropy from the supercooling process of EWPT for the two-step pattern is evaluated by [31]

$$
\Delta s=-\left(\left.\frac{d V}{d T}\right|_{\phi}-\left.\frac{d V}{d T}\right|_{H}\right)
$$

with the finite temperature potential $V$ included only the thermal mass correction evaluated at the nucleation temperatures, which has been normalized by the SM entropy in the radiation-dominated Universe,

$$
s=\frac{2 \pi^{2}}{45} g_{\star s} T_{\mathrm{EW}}^{3}
$$

By taking $g_{\star s}=100$ there, it was estimated that $\Delta s / s_{\mathrm{EW}}$ is around the percent level around the phase transition temperature $T_{n}$ for benchmarks of $O \rightarrow A(B) \rightarrow C$. As in the standard model (see Ref. [84]), one finds a negligible dilution factor, namely,

$$
\left(\frac{a_{f}}{a_{i}}\right)^{3}=\frac{s_{+}}{s_{-}} \approx 1+\frac{\Delta s}{s_{+}} \approx 1.01
$$

with the entropy difference between the high-temperature and low-temperature entropy: $\Delta s=s_{+}-s_{-}$.
[1] P. A. R. Ade et al. (Planck Collaboration), Astron. Astrophys. 594, A13 (2016).

[2] G. Bertone and D. Hooper, Rev. Mod. Phys. 90, 045002 (2018).

[3] A. Tan et al. (PandaX-II Collaboration), Phys. Rev. Lett. 117, 121303 (2016).

[4] E. Aprile et al. (XENON Collaboration), Phys. Rev. Lett. 119, 181301 (2017).

[5] D. S. Akerib et al. (LUX Collaboration), Phys. Rev. Lett. 118, 021303 (2017).

[6] A. Albert et al. (Fermi-LAT and DES Collaborations), Astrophys. J. 834, 110 (2017).

[7] H. Abdallah et al. (H.E.S.S. Collaboration), Phys. Rev. Lett. 117, 111301 (2016).

[8] H. Abdalla et al. (H.E.S.S. Collaboration), Phys. Rev. Lett. 117, 151302 (2016).

[9] A. Abramowski et al. (H.E.S.S. Collaboration), Phys. Rev. Lett. 114, 081301 (2015).

[10] M. Aguilar et al. (AMS Collaboration), Phys. Rev. Lett. 117, 091103 (2016).

[11] H. Baer, K. Y. Choi, J. E. Kim, and L. Roszkowski, Phys. Rep. 555, 1 (2015).

[12] G. Arcadi, M. Dutra, P. Ghosh, M. Lindner, Y. Mambrini, M. Pierre, S. Profumo, and F. S. Queiroz, Eur. Phys. J. C 78, 203 (2018).
[13] J. McDonald, Phys. Rev. Lett. 88, 091304 (2002).

[14] L. J. Hall, K. Jedamzik, J. March-Russell, and S. M. West, J. High Energy Phys. 03 (2010) 080.

[15] N. Bernal, M. Heikinheimo, T. Tenkanen, K. Tuominen, and V. Vaskonen, Int. J. Mod. Phys. A 32, 1730023 (2017).

[16] T. Tenkanen and V. Vaskonen, Phys. Rev. D 94, 083516 (2016).

[17] M. Shaposhnikov and I. Tkachev, Phys. Lett. B 639, 414 (2006).

[18] F. Bezrukov, D. Gorbunov, and M. Shaposhnikov, J. Cosmol. Astropart. Phys. 06 (2009) 029.

[19] P. S. Bhupal Dev, A. Mazumdar, and S. Qutub, Front. Phys. 2, 26 (2014).

[20] L. Kofman, A. D. Linde, and A. A. Starobinsky, Phys. Rev. Lett. 73, 3195 (1994).

[21] P. Adshead, Y. Cui, and J. Shelton, J. High Energy Phys. 06 (2016) 016.

[22] M. Blennow, E. Fernandez-Martinez, and B. Zaldivar, J. Cosmol. Astropart. Phys. 01 (2014) 003.

[23] H. M. Hodges, Phys. Rev. D 47, 456 (1993).

[24] Z. G. Berezhiani, A. D. Dolgov, and R. N. Mohapatra, Phys. Lett. B 375, 26 (1996).

[25] S. Dodelson and L. M. Widrow, Phys. Rev. Lett. 72, 17 (1994). 
[26] M. J. Baker and J. Kopp, Phys. Rev. Lett. 119, 061801 (2017).

[27] M. J. Baker, M. Breitbach, J. Kopp, and L. Mittnacht, J. High Energy Phys. 03 (2018) 114.

[28] L. Bian and Y. L. Tang, J. High Energy Phys. 12 (2018) 006.

[29] A. Hektor, K. Kannike, and V. Vaskonen, Phys. Rev. D 98, 015032 (2018).

[30] N. Arkani-Hamed, T. Han, M. Mangano, and L. T. Wang, Phys. Rep. 652, 1 (2016).

[31] H. H. Patel and M. J. Ramsey-Musolf, Phys. Rev. D 88, 035013 (2013).

[32] W. Cheng and L. Bian, Phys. Rev. D 98, 023524 (2018).

[33] D. Curtin, P. Meade, and C. T. Yu, J. High Energy Phys. 11 (2014) 127.

[34] W. Chao, H. K. Guo, and J. Shu, J. Cosmol. Astropart. Phys. 09 (2017) 009.

[35] M. Pospelov, A. Ritz, and M. B. Voloshin, Phys. Lett. B 662, 53 (2008).

[36] A. Berlin, D. Hooper, and G. Krnjaic, Phys. Lett. B 760, 106 (2016).

[37] P. F. De Salas, S. Gariazzo, O. Mena, C. A. Ternes, and M. Tórtola, Front. Astron. Space Sci. 5, 36 (2018).

[38] N. Aghanim et al. (Planck Collaboration), arXiv:1807.06209.

[39] Y. Farzan and E. Ma, Phys. Rev. D 86, 033007 (2012).

[40] X. Liu and L. Bian, Phys. Rev. D 97, 055028 (2018).

[41] E. Molinaro, C. E. Yaguna, and O. Zapata, J. Cosmol. Astropart. Phys. 07 (2014) 015.

[42] E. Ma, Phys. Rev. D 73, 077301 (2006).

[43] K. S. Babu and E. Ma, Int. J. Mod. Phys. A 23, 1813 (2008).

[44] W. Cheng and L. Bian, arXiv:1805.00199 [Phys. Rev. D (to be published)].

[45] N. Blinov, S. Profumo, and T. Stefaniak, J. Cosmol. Astropart. Phys. 07 (2015) 028.

[46] A. G. Hessler, A. Ibarra, E. Molinaro, and S. Vogl, J. High Energy Phys. 01 (2017) 100.

[47] H. H. Patel and M. J. Ramsey-Musolf, J. High Energy Phys. 07 (2011) 029.

[48] I. Affleck, Phys. Rev. Lett. 46, 388 (1981).

[49] A. D. Linde, Nucl. Phys. B216, 421 (1983); B223, 544 (1983).

[50] A. D. Linde, Phys. Lett. 100B, 37 (1981).

[51] http://pdg.lbl.gov/2017/reviews/rpp2017-rev-bbang-nucleosynthesis.pdf.

[52] C. Caprini et al., J. Cosmol. Astropart. Phys. 04 (2016) 001.

[53] R. G. Cai, Z. Cao, Z. K. Guo, S. J. Wang, and T. Yang, Natl. Sci. Rev. 4, 687 (2017).

[54] A. Kosowsky, M. S. Turner, and R. Watkins, Phys. Rev. D 45, 4514 (1992).

[55] A. Kosowsky, M. S. Turner, and R. Watkins, Phys. Rev. Lett. 69, 2026 (1992).

[56] A. Kosowsky and M. S. Turner, Phys. Rev. D 47, 4372 (1993).
[57] S. J. Huber and T. Konstandin, J. Cosmol. Astropart. Phys. 09 (2008) 022.

[58] M. Kamionkowski, A. Kosowsky, and M. S. Turner, Phys. Rev. D 49, 2837 (1994).

[59] A. Beniwal, M. Lewicki, J. D. Wells, M. White, and A. G. Williams, J. High Energy Phys. 08 (2017) 108.

[60] G. Kurup and M. Perelstein, Phys. Rev. D 96, 015036 (2017).

[61] F. P. Huang, Z. Qian, and M. Zhang, Phys. Rev. D 98, 015014 (2018).

[62] V. Vaskonen, Phys. Rev. D 95, 123515 (2017).

[63] J. Ellis, M. Lewicki, and J. M. No, arXiv:1809.08242.

[64] A. Addazi and A. Marciano, Chin. Phys. C 42, 023105 (2018).

[65] A. Addazi, Y. F. Cai, and A. Marciano, Phys. Lett. B 782, 732 (2018).

[66] Q. H. Cao, F. P. Huang, K. P. Xie, and X. Zhang, Chin. Phys. C 42, 023103 (2018).

[67] F. P. Huang, P. H. Gu, P. F. Yin, Z. H. Yu, and X. Zhang, Phys. Rev. D 93, 103515 (2016).

[68] M. Carena, Z. Liu, and M. Riembau, Phys. Rev. D 97, 095032 (2018).

[69] D. Goncalves, T. Han, and S. Mukhopadhyay, Phys. Rev. Lett. 120, 111801 (2018); 121, 079902(E) (2018).

[70] D. E. Morrissey and M. J. Ramsey-Musolf, New J. Phys. 14, 125003 (2012).

[71] B. Grzadkowski and D. Huang, J. High Energy Phys. 08 (2018) 135.

[72] M. Jiang, L. Bian, W. Huang, and J. Shu, Phys. Rev. D 93, 065032 (2016).

[73] D. Borah, P.S. B. Dev, and A. Kumar, arXiv:1810 .03645 .

[74] S. Baumholzer, V. Brdar, and P. Schwaller, J. High Energy Phys. 08 (2018) 067.

[75] D. Borah, A. Dasgupta, and S. K. Kang, arXiv:1806.04689.

[76] T. Hugle, M. Platscher, and K. Schmitz, Phys. Rev. D 98, 023020 (2018).

[77] K. Kannike, Eur. Phys. J. C 72, 2093 (2012).

[78] R. Barbieri, L. J. Hall, and V. S. Rychkov, Phys. Rev. D 74, 015007 (2006).

[79] E. Lundstrom, M. Gustafsson, and J. Edsjo, Phys. Rev. D 79, 035013 (2009).

[80] A. Pierce and J. Thaler, J. High Energy Phys. 08 (2007) 026.

[81] A. Belyaev, N. D. Christensen, and A. Pukhov, Comput. Phys. Commun. 184, 1729 (2013).

[82] A. Alloul, N. D. Christensen, C. Degrande, C. Duhr, and B. Fuks, Comput. Phys. Commun. 185, 2250 (2014).

[83] C. Wainwright and S. Profumo, Phys. Rev. D 80, 103517 (2009).

[84] M. Quiros, arXiv:hep-ph/9901312. 